(C) 2000 International Press

Adv. Theor. Math. Phys. 4 (2000) 1259-1319

\title{
On the General Structure of the
}

\section{Non-Abelian Born-Infeld Action}

\author{
L. Cornalba \\ École Normale Supérieure \\ Paris, France \\ cornalba@lpt.ens.fr
}

\begin{abstract}
We discuss the general structure of the non-abelian Born-Infeld action, together with all of the $\alpha^{\prime}$ derivative corrections, in flat $D$ dimensional space-time. More specifically, we show how the connection between open strings propagating in background magnetic fields and gauge theories on non-commutative spaces can be used to constrain the form of the effective action for the massless modes of open strings at weak coupling. In particular, we exploit the invariance in form of the effective action under a change of non-commutativity scale of spacetime to derive algebraic equations relating the various terms in the $\alpha^{\prime}$ expansion. Moreover, we explicitly solve these equations in the simple case $D=2$, and we show, in particular, how to construct the minimal invariant derivative extension of the NBI action.
\end{abstract}

\section{Introduction}

The dynamics of massless open string states propagating in flat space-time can be described, at weak string coupling, by an effective action

e-print archive: http://xxx.lanl.gov/hep-th/0006018 


$$
S(A) \sim \frac{1}{g_{s}} \int \operatorname{Tr} \mathcal{L}(A)
$$

which is function of a $U(N)$ gauge potential $A$ and where the lagrangian density $\mathcal{L}$ is, in general, an expansion in $\alpha^{\prime}$ written in terms of arbitrary powers of the curvature $F$ and of its covariant derivatives. The action $S$ has various equivalent interpretations. First of all, it reproduces, at tree level, the disk amplitudes computed directly in string theory. Secondly, the equations of motion derived from $S$ correspond to the condition of conformal invariance of the open string sigma model with Wilson line interactions $\operatorname{Tr} P \exp \left(i \int_{\partial \Sigma} A\right)$ on the boundary $\partial \Sigma$ of the string world-sheet. In fact, it was shown by Tseytlin and Andreev [4] that the effective action itself can be identified with the partition function of the $\sigma$-model. Finally we recall that, using $T$-duality, the action $S(A)$ also describes the weak coupling dynamics of $D$-branes of all dimensions.

New progress in the understanding of the general form of the effective action $S$ has been made in a recent work by Seiberg and Witten. In [1] the authors show that, if the open string propagates in a space-time with a constant background NSNS two-form field $B$, then the dynamics of the open-string modes can be described in two equivalent ways. Firstly, one can consider the original action $S$, and add to the curvature $F$ a central term $B \cdot 1$. Alternatively, one can replace the original $U(N)$ gauge theory by a gauge theory on a non-commutative space, with non-commutativity parameter $\theta$ related to the background metric $g$ and two-form $B$. The action then takes the same form as the original action $S$, with the gauge potential $A$ replaced with the gauge potential $\widehat{A}$ of a non-commutative gauge theory, and with products of fields replaced with Moyal products $\star$ with parameter $\theta$. More conjecturally, it is shown in [1] that one can in fact choose the parameter $\theta$ freely by properly adjusting the central term $\Phi \cdot \mathbf{1}$ added to the non-commutative curvature $\widehat{F}$. For each value of $\theta$, the action describing the open-string dynamics has then the same exact form as the original action $S$. The parameter $\theta$ is then a redundant parameter, since different values of $\theta$ correspond to the same underlying physics. On the other hand, the simple fact that the form of the action at various values of $\theta$ is invariant imposes severe restrictions on the possible structure of the original action $S$. This paper is devoted to the understanding of those restrictions. Previous work on this subject is contained in $[10,11]$.

Before describing the results let us remark a basic fact. In order to attack the problem of invariance, one need not consider the full $U(N)$ theory. In fact, if one restricts the attention to the $U(1)$ case, but considers non-zero 
values of the non-commutativity parameter $\theta$, one is then considering a theory which is effectively non-abelian. From an algebraic point of view, the requirements imposed by form-invariance of the action are identical in the $U(1)$ and $U(N)$ case as soon as $\theta \neq 0$. For this reason we work throughout in the $U(1)$ case, but the results will, at the end, be valid in the general $U(N)$ setting. It is really quite remarkable that the simpler abelian theory contains, in an subtle way indeed, the complete information about the general nonabelian theory.

Let us now describe the general results of this paper. As just remarked, they are valid in the general non-abelian $U(N)$ setting. An invariant action $S$ is given as a linear combination $S=\sum_{i} c_{i} I_{i}$ of basic actions $I_{i}$, which we call invariant blocks. A single block $I$ has itself the following structure. First write $I$ as an expansion in $\alpha^{\prime}$, as

$$
I \propto \sum_{L \geq P}\left(\alpha^{\prime}\right)^{L} I_{L}
$$

where we call a term proportional to $\left(\alpha^{\prime}\right)^{L}$ a term of level $L$. The lowest level term $I_{P}$ is a pure derivative term. The precise meaning of this notion will be given later in the paper, but informally we can say that pure derivative terms are those which are invariant under addition of a central term to the curvature $F$ (the basic example is the $F^{2}$ term at level $P=2$ ). The higher level terms $I_{L}$ are then needed in order to achieve invariance of the full action $I$ under a change of the parameter $\theta$. The basic result of this paper is to reduce the question of invariance to a set of algebraic equations relating the various terms $I_{L}$. In particular, we will show that the requirement of invariance can be rephrased in terms of four basic algebraic operators

$$
\begin{array}{llll}
\Delta & \bar{\Delta} & \delta & \bar{\delta}
\end{array}
$$

which depend on an arbitrary antisymmetric matrix $\Delta_{a b}$ and which satisfy the basic commutation relations

$$
[\bar{\Delta}, \Delta]=[\delta, \bar{\delta}]=2 L-\frac{D}{2},
$$

where $D$ is the dimension of space-time. The various terms $I_{L}$ then must satisfy the equations

$$
\begin{aligned}
\Delta I_{L} & =\delta I_{L+1} \\
\bar{\Delta} I_{L+1} & =\bar{\delta} I_{L} .
\end{aligned}
$$

A term of lowest level satisfies then $\Delta I_{P}=\bar{\delta} I_{P}=0$. 
We also show in this paper how the above equations can be explicitly solved in $D=2$. This is clearly a toy model, since gauge-bosons in two dimensions do not have propagating degrees of freedom. On the other hand, the algebraic equations are perfectly well defined in dimension 2 , and are highly non-trivial. This model is useful for a variety of reasons. First of all, one can show that, starting from the lowest level $F^{2}$ term, one can reconstruct the full $\mathrm{BI}$ action, plus a minimal set of derivative corrections which are required for invariance of the action (we show that derivative corrections already enter at level 4). Similarly, one expects that the invariant block built from $F^{2}$ in general dimension $D$ will be the minimal derivative extension of the NBI action. Finally, the construction in $D=2$ is a first indication of how to solve the equations (2) in the general case.

We have said that the action $S$ is a linear combination of invariant blocks. The specific coefficients are not constrained by the methods of this paper, and should be determined by other means. However let us note that none of the arguments which follow rely on supersymmetry, and therefore we expect supersymmetry to impose constraints on the coefficients themselves, restricting even more the set of allowed forms of the action.

We shall now describe the contents of this paper. First let me note that section 5.2 contains a concise summary of the results, which includes the main equations in the text. The structure of the paper is as follows. In section 2 we review the general structure of the action $S$, as can be inferred from the analysis of scattering amplitudes of gauge bosons. We then use this knowledge in section 3 to rewrite the effective action as a matrix action. In doing so, we review the general form of the Seiberg-Witten map which relates commutative and non-commutative gauge potentials, and we introduce the formal algebraic machinery which is required in the sequel of the paper. We also derive the second equation in (2). Section 4 is then devoted to rewriting the results of section 3 in an invariant operator language. The purpose is two-fold. On one side, the structure of the action becomes more transparent. Moreover, in this setting, the question of invariance from $\theta$ is more easily understood and solved. Section 5 then applies the results of section 4 to the specific problem at hand, and completes the derivation of the equations (2), together with the basic commutator (1). Section 6 is then devoted to the explicit solution of equations (2) in the case of $D=2$. We conclude in section 7 with discussion and comments on open problems for future research.

The results of this paper require, together with the general discussion, a considerable number of technical lemmata. We have tried to limit the technical discussion to a minimum in the main body of the paper, leaving the precise proofs of many statements to a rather large appendix. 


\section{The General Form of the Effective Action}

We consider an open string propagating in flat $D$-dimensional space-time $M$ with coordinates $x^{a}$ and with constant metric $g_{a b}$. Throughout the paper we use units such that

$$
2 \pi \alpha^{\prime}=1
$$

We concentrate on the physics of the massless $U(N)$ gauge bosons, to lowest order in the string coupling constant $g_{s}$. The amplitude $\mathcal{A}\left(p^{I}\right)$ for the scattering of $n$ gluons with momenta $p^{I}$ - with $g^{a b} p_{a}^{I} p_{b}^{I}=0$ - is computed starting from the disk $n$-point function of the corresponding vertex operators, cyclically ordered on the boundary of the string world-sheet, and then by summing over the cyclically inequivalent orderings. More precisely, one has that

$$
\mathcal{A}\left(p^{I}\right) \sim g_{s}^{n-2} \sum_{\substack{\sigma \text { cyclically } \\ \text { inequivalent }}} \mathcal{F}_{g}\left(p^{\sigma_{1}}, \cdots, p^{\sigma_{n}}\right)
$$

where $\mathcal{F}_{g}$ depends on the metric $g$ and is invariant under cyclic permutations of the arguments ${ }^{1}$.

One can equivalently summarize the information about disk amplitudes by introducing an effective action $S(A)$, function of a $U(N)$ connection $A$ on $M$, such that the tree level amplitudes of $S$ are equal to the disk amplitudes $\mathcal{A}$. The general form of the action $S$ is well known and reads

$$
S\left(A, g, g_{s}\right)=\frac{\operatorname{Tr}}{g_{s}} \int d^{D} x \operatorname{det}^{\frac{1}{2}} g_{a b}\left(1+\frac{1}{4} F_{a b} F_{c d} g^{a c} g^{b d}+\cdots\right) .
$$

We have absorbed any numerical prefactor in the definition of $g_{s}$. Moreover, the terms hidden in $\cdots$ contain both higher powers of the field strength and derivative terms ${ }^{2}$. As a note on conventions, in all that follows actions will always be written assuming a Euclidean signature of the metric.

\footnotetext{
${ }^{1} \mathrm{We}$ omit explicit reference to polarizations.

${ }^{2}$ Various facts are known about the terms in (3). First of all, in the $U(1)$ case, the terms without derivatives resum to the Born-Infeld lagrangian $[14,12]$

$$
S(A)=\frac{1}{g_{s}} \int d^{D} x \operatorname{det}^{\frac{1}{2}}(g+F) .
$$

In the non-abelian case even the non-derivative terms are not completely known. At order $F^{4}$ the computation can be explicitly carried out in string theory, and the result is proportional to [5]

$$
\operatorname{Tr}\left(F_{a b} F_{c b} F_{a d} F_{c d}+\frac{1}{2} F_{a b} F_{c b} F_{c d} F_{a d}-\frac{1}{4} F_{a b} F_{a b} F_{c d} F_{c d}-\frac{1}{8} F_{a b} F_{c d} F_{a b} F_{c d}\right) .
$$
}


Let us now introduce a constant background NS-NS two-form field $B_{a b}$. In the presence of open strings, a constant field $B$ is not pure gauge and does affect the dynamics of the gauge bosons. In particular, the effects have been very clearly analyzed in $[1,2]$ and can be summarized as follows

- The momenta of the asymptotic gluon states satisfy a modified on-shell condition. More precisely, gluons are massless with respect to an effective open string metric $G=g-B \frac{1}{g} B$ and therefore the corresponding momenta satisfy $G^{a b} p_{a} p_{b}=0$.

- The effective coupling constant is modified to an open string value of $G_{s}=g_{s} \operatorname{det}^{\frac{1}{2}} G \operatorname{det}^{-\frac{1}{2}}(g+B)$

- Finally, the disk scattering amplitudes are modified by momentum dependent phase factors. In particular, in terms of the antisymmetric matrix $\theta^{-1}=B-g \frac{1}{B} g$, the amplitudes are given by

$$
\mathcal{A}\left(p^{I}\right) \sim G_{s}^{n-2} \sum_{\substack{\sigma \text { cyclically } \\ \text { inequivalent }}} \mathcal{F}_{G}\left(p^{\sigma_{1}}, \cdots, p^{\sigma_{n}}\right) e^{-\frac{i}{2} \theta^{a b} \sum_{I>J} p_{a}^{\sigma_{I}} p_{b}^{\sigma_{J}}}
$$

The above dynamics can again be summarized in tree diagrams of a modified effective action which can be written, starting from (3), in various different but equivalent ways . Let me briefly review the various options:

At higher orders in $F$, the more reasonable proposal is a natural extension of the BornInfeld action proposed by Tseytlin [5] in terms of a symmetrized trace prescription

$$
S(A)=\frac{\mathrm{STr}}{g_{s}} \int d^{D} x \operatorname{det}^{\frac{1}{2}}(g+F) .
$$

The above prescription not only matches (up to order $F^{4}$ ) with the scattering computations in superstring theory, but also matches results for $D$-brane actions derived within matrix theory. $[17,18]$. On the other hand, it is known that the symmetrized trace prescription is incomplete at order $F^{6}$. In [19], the authors study the spectra of excitations around diagonal and intersecting $\mathrm{D}$-brane configurations on tori, and find discrepancies with the prescription (4). The correction terms at order $\alpha^{\prime 3}$ have been explicitly computed in [20].

Some derivative corrections are known, both in the $U(1)$ case, as well as in the nonabelian setting. For the $U(1)$ theory, some derivative terms have been computed [4]. In particular, for bosonic open string theory, the authors find terms at order $F^{2} \partial F \partial F$. Still in [4], derivative correction in superstring theory at order $F^{2} \partial \partial F \partial \partial F$ are discussed. In [21], the author finds derivative corrections at order $F^{5}$ (and $F^{3} D^{2} F$ ), proportional to $\zeta(3)$, by studying 5 -point disk amplitudes. Finally, in the bosonic theory, there is a known derivative term at order $F^{3}$ which is proportional to

$$
\operatorname{Tr}\left(F_{a b}\left[F_{b c}, F_{c a}\right]\right) .
$$


1. On one hand, one can follow the usual prescription by starting with the action (3) and by simply adding to the field strength $F$ the central term $B \cdot \mathbf{1}$.

2. On the other hand, one can follow the ideas of [1]. In this case, one considers the action (3) with the replacements $g_{a b} \rightarrow G_{a b}$ and $g_{s} \rightarrow$ $G_{s}$. This correctly reproduces the modified gauge coupling and the modified mass-shell condition. Phase factors in (5) are reproduced by reinterpreting the matrix $\theta$ as a non-commutative scale of spacetime, and substituting, in the action (3), ordinary products of fields with Moyal products in terms of $\theta$. Correspondingly, the $U(N)$ gauge potential $A$ is now mapped into a gauge potential

$$
\widehat{A}=\widehat{A}_{S W}(A, \theta)
$$

of a $U(N)$ non-commutative gauge theory, and the gauge group is modified accordingly. We will call the map $\widehat{A}_{S W}$ the Seiberg-Witten map.

NOTATION. We have denoted in (3) by $S\left(A, g, g_{s}\right)$ the effective action at zero $B$ field, as a function of the gauge potential, the metric, and the coupling constant. At finite $B$, there are two new relevant parameters in the description of the action - a possible central term added to the curvature, and a possible non-commutativity parameter. In general, the action will then depend on five parameters

$$
S \text { (potential, metric, coupling, central term, NC paramter). }
$$

Then the equivalence of the descriptions 1 and 2 above is just

$$
S\left(A, g, g_{s}, B, 0\right)=S\left(\widehat{A}, G, G_{s}, 0, \theta\right) .
$$

3. Finally, one can follow a naive procedure, which is usually not considered, but which will be important for our future discussion. In fact, this procedure is the most natural one if we are given only the information about the amplitudes (5), without any reference to an underlying string theory. Specifically, we may wish to reproduce directly the amplitudes (5) using a standard $U(N)$ theory, without using any previous information about the theory at zero $B$. Firstly, the kinetic term of the theory must be

$$
\frac{\operatorname{Tr}}{G_{s}} \int d^{D} x \operatorname{det}^{\frac{1}{2}} G_{a b}\left(\frac{1}{4} G^{a c} G^{b d} F_{a b} F_{c d}\right)
$$


in order to reproduce the correct mass-shell condition. We can write the above equation in a more suggestive form by introducing the matrix $\Gamma=g+B$ and by first noting that, since $\operatorname{Tr}(F \wedge F)$ is a total derivative, then

$$
\int d^{D} x \operatorname{Tr}\left(F_{a b} F_{c d}+F_{a c} F_{d b}+F_{a d} F_{b c}\right)=0
$$

This identity, together with the facts that $2 G^{a b}=\Gamma^{a b}+\Gamma^{b a}$ and that $G_{s}^{-1} \operatorname{det}^{\frac{1}{2}} G=g_{s}^{-1} \operatorname{det}^{\frac{1}{2}} \Gamma$, can be used to show that the kinetic term (6) is equal to

$$
\frac{\operatorname{Tr}}{g_{s}} \int d^{D} x \operatorname{det} \frac{1}{2} \Gamma_{a b}\left(\frac{1}{4} \Gamma^{a c} \Gamma^{d b} F_{a b} F_{c d}+\frac{1}{8} \Gamma^{a b} F_{a b} \Gamma^{c d} F_{c d}\right) .
$$

The above is nothing but the quadratic term coming from the expansion of the Born-Infeld action $\sqrt{g+B+F}=\sqrt{\Gamma+F}$. The matrix $\Gamma$, which is not symmetric, now plays the role of the metric and therefore more tensor structures are possible (in fact, the expansion of the $\mathrm{BI}$ action includes all powers of $F$, including the odd ones). In some sense, we have traded the central term $B \cdot \mathbf{1}$ added to the curvature $F$ with an addition to the metric $g \rightarrow g+B$, by allowing metrics to be non-symmetric. This invariance is natural from the point of view of the open string $\sigma$-model

$$
\int_{\Sigma} g_{a b} \partial X^{a} \bar{\partial} X^{b}+\int_{\Sigma} B+\int_{\partial \Sigma} A
$$

where we see, using $\int_{\partial \Sigma} A=\int_{\Sigma} F$, that only the combination $g+B+F$ has an invariant meaning. We are then led to conclude that there is an extension of (3) in the case of a non-symmetric metric $\Gamma$ of the general form

$$
\frac{\operatorname{Tr}}{g_{s}} \int d^{D} x \operatorname{det} \frac{1}{2} \Gamma_{a b}\left[1-\frac{1}{2} \Gamma^{a b} F_{a b}+\cdots\right]
$$

which reproduces the amplitudes (5). Moreover, we claim that all contractions of indices in $\cdots$ are done with $\Gamma^{a b}$ (no terms containing $\Gamma_{a b}$ ). This fact can be shown starting with (5). In fact, these amplitudes are just functions of $G^{-1}$ and $\theta$, which only depend on $\Gamma^{a b}$. This shows that the amplitudes do not depend on $\Gamma_{a b}$. To show the same fact for the vertices of the action, we must show that the subtractions coming from poles in the various subchannels also share the same property. The only problems could come from internal propagators $p^{-2} G_{a b}$. We recall though that [1], for amplitudes of the form (5), a general tree 
graph is computed by first analyzing the graph at $\theta=0$, and then by multiplying it by a phase factor depending only on external momenta. Moreover, the graph at $\theta=0$ has all propagators $p^{-2} G_{a b}$ contracted with metrics $G^{a b}$ on the vertices, thus proving that the subtraction is again just a function of $G^{-1}$ and $\theta$. Using the general form (7) of the action we can then give a meaning to the function $S$ when the metric is not symmetric. We can then summarize the equality of descriptions 1 and 3 by saying that

$$
S\left(A, g, g_{s}, B, 0\right)=S\left(A, g+B, g_{s}, 0,0\right) .
$$

We have then seen that we can trade a central term $B \cdot \mathbf{1}$ with either a non-commutativity parameter $\theta$ or with an addition $g \rightarrow g+B$ to the metric. It is natural (following $[1,15]$ ) to conjecture that, in fact, one has a continuous family of possibilities, parametrized by a central term $\Phi \cdot \mathbf{1}$ and by a free parameter $\theta$. The effective non-symmetric metric $\Gamma$ then combines with the central term $\Phi$ into the invariant combination $\Gamma+\Phi$, which, following again [1], is given by

$$
\frac{1}{\Gamma+\Phi}+\theta=\frac{1}{g+B}
$$

The effective coupling again depends only on the sum $\Gamma+\Phi$, and is given by

$$
\frac{1}{G_{s}} \operatorname{det}^{\frac{1}{2}}(\Gamma+\Phi)=\frac{1}{g_{s}} \operatorname{det}^{\frac{1}{2}}(g+B) .
$$

Finally, the gauge potential is given by the Seiberg-Witten map $\widehat{A}=\widehat{A}_{S W}(A, \theta)$. The action $S\left(\widehat{A}, \Gamma, G_{s}, \Phi, \theta\right)$ is then independent of $\Phi$ and $\theta$.

\section{The Effective Action as a Matrix Action}

In this section we continue our general analysis of the effective action $S$, but we restrict our attention to the $U(1)$ case. As already noted in the introduction, whenever the non-commutativity scale $\theta$ is non-zero, the $U(1)$ case contains the physics of the full $U(N)$ theory, and we therefore lose nothing in concentrating on the effective action for $N=1$.

\subsection{Choosing the central term}

In the previous section we have argued that the effective action describing the dynamics of gluons in space-time is given by a function $S\left(\widehat{A}, \Gamma, G_{s}, \Phi, \theta\right)$ of 
five arguments - i.e. the gauge potential $\widehat{A}$, the generalized non-symmetric metric $\Gamma$, the coupling $G_{s}$, the central term $\Phi$ and the non-commutativity parameter $\theta$.

The arguments of the action $S$ are not all independent, since physically different backgrounds are parametrized only by the closed string parameters $A, g+B$ and $g_{s}$, which we keep fixed. In fact, the same physical situation corresponds to a family of different values of the arguments of $S$, parameterized by $\Phi$ and $\theta$, which we consider as free parameters. The remaining variables $\widehat{A}, \Gamma$ and $G_{s}$ are then determined, in terms of the fixed closed string parameters, by the equations

$$
\begin{aligned}
\widehat{A} & =\widehat{A}_{S W}(A, \theta) \\
\frac{1}{\Gamma+\Phi}+\theta & =\frac{1}{g+B} \\
\frac{1}{G_{s}} \operatorname{det}^{\frac{1}{2}}(\Gamma+\Phi) & =\frac{1}{g_{s}} \operatorname{det}^{\frac{1}{2}}(g+B) .
\end{aligned}
$$

The action $S$ is then independent of $\Phi$ and $\theta$.

We use this freedom to choose the central term $\Phi$. Throughout the paper we will denote with $K$ the inverse of $\theta$

$$
K=\frac{1}{\theta}
$$

Using the independence of $S$ on $\Phi$, we set

$$
\Phi=-K
$$

The only free parameter is then the non-commutativity scale $\theta$.

Equation (8) can be easily rewritten in terms of the combination

$$
\gamma=g+B-K
$$

and reads

$$
\Gamma=-K \frac{1}{\gamma} K
$$

Finally equation (9), which determines the coupling, becomes

$$
\frac{1}{G_{s}} \operatorname{det}^{\frac{1}{2}} \Gamma=\frac{1}{g_{s}} \operatorname{det}^{\frac{1}{2}} K
$$




\subsection{A matrix action}

Let us now consider the expression for the field strength $\widehat{F}$ and its covariant derivatives. We start by introducing the coordinate functions

$$
x_{a}=K_{a b} x^{b}
$$

and the combinations

$$
\begin{aligned}
\lambda_{a} & =x_{a}+\widehat{A}_{a} \\
\lambda^{a} & =\theta^{a b} \lambda_{b}=x^{a}+\theta^{a b} \widehat{A}_{b} .
\end{aligned}
$$

Note that we raise and lower indices with the matrix $\theta, K$. Using the simple fact that, for any function $f$,

$$
\partial_{a} f=-i\left[x_{a}, f\right],
$$

we quickly see that the commutator $-i\left[\lambda_{a}, \lambda_{b}\right]$ is given by

$$
\begin{aligned}
-i\left[\lambda_{a}, \lambda_{b}\right] & =\widehat{F}_{a b}-K_{a b} \\
& =\widehat{F}_{a b}+\Phi_{a b}
\end{aligned}
$$

and therefore computes the field strength with the addition of the correct central term. Moreover, for any function $f$ which transforms in the adjoint representation of the non-commutative gauge group, the commutator

$$
\begin{aligned}
-i\left[\lambda_{a}, f\right] & =\partial_{a} f-i\left[\widehat{A}_{a}, f\right] \\
& =\widehat{D}_{a} f
\end{aligned}
$$

computes the covariant derivative $\widehat{D}_{a} f$. Therefore, any expression involving products of covariant derivatives of the field strength, with the addition of the central term $\Phi=-K$, can be expressed in terms of $\star$ products of the functions $\lambda_{a}$-for example, an expression like $(\widehat{F}+\Phi)_{a b} \star \widehat{D}_{c}(\widehat{F}+\Phi)_{d e}$ can be rewritten as $i\left[\lambda_{a}, \lambda_{b}\right] \star\left[\lambda_{c},\left[\lambda_{d}, \lambda_{e}\right]\right]$. We conclude that the general form of the effective action $S$ is

$$
\frac{1}{G_{s}} \sum_{n \text { even }} \int d^{D} x \operatorname{det}^{\frac{1}{2}} \Gamma\left(\lambda_{a_{1}} \star \cdots \star \lambda_{a_{n}}\right) \eta^{a_{1} \cdots a_{n}}
$$

where the coefficients $\eta^{a_{1} \cdots a_{n}}$ are constructed from the matrix $\Gamma^{a b}$. We may further manipulate the above equation using (10) and (11) and raising and lowering indices with the matrix $\theta, K$. We can then write

$$
S=\frac{1}{g_{s}} \sum_{n \text { even }} \int d^{D} x \operatorname{det}^{\frac{1}{2}} K\left(\lambda^{1} \star \cdots \star \lambda^{n}\right) \eta_{1 \cdots n}
$$


We have used a compact notation for indices, which will be used extensively in the sequel, substituting $a_{1} \rightarrow 1, a_{2} \rightarrow 2, \cdots$. Moreover, the symbol $\eta_{1 \ldots n}$ represents the tensor which is built from the matrix $\gamma_{a b}$ exactly as $\eta^{1 \cdots n}$ is constructed starting from $\Gamma^{a b}$. For example, if $\eta^{1234}=\Gamma^{13} \Gamma^{24}$ then $\eta_{1234}=\gamma_{13} \gamma_{24}$.

REMARK . Let us recall that, in the general $U(N)$ effective action, the distinction between derivative and non-derivative terms is ambiguous, since a commutator of covariant derivatives $\left(D_{a} D_{b}-D_{b} D_{a}\right) \cdots$ is equivalent to a commutator with the field strength $\left[F_{a b}, \cdots\right]$. In fact, when one writes the action in matrix form as in (12), all terms (derivative and non-derivative) are included on an equal footing. In particular, the ambiguity discussed above becomes naturally the Jacobi identity of the commutator $\left[\lambda^{a}, \lambda^{b}\right]$.

\subsection{Gauge invariance and invariance under addition of a cen- tral term}

In the previous subsection we have rewritten the action $S$ in the compact form (12), which is more suited for discussing the action in its entirety, including terms with arbitrary powers of the field strength and with arbitrary number of derivatives. On the other hand, the gauge invariance of the original action is not immediately transparent in this new notation, and one needs to restate the requirement of gauge invariance in terms of the tensors $\eta_{1 \cdots n}$. This is easily done by noting that the functions $\lambda_{a}$, when used in covariant expressions, always appear within commutators, and therefore adding a constant $\varepsilon_{a}$ to the function $\lambda_{a}$ does not change the action. Gauge invariance becomes then, within the matrix formulation (12) of the action, invariance under translations $\lambda^{a} \rightarrow \lambda^{a}+\varepsilon^{a}$. This requirement quickly translates into the following algebraic relation which must be satisfied by the tensors $\eta$

$$
\eta_{123 \cdots n}+\eta_{213 \cdots n}+\eta_{231 \cdots n}+\cdots+\eta_{23 \cdots 1 n}+\eta_{23 \cdots n 1}=0 .
$$

We will call tensors satisfying the above equation gauge invariant (GI).

In order to rewrite the effective action as a matrix action, we had fixed, in section 3.2 , the central term $\Phi$ to $-K$. We must then require by hand that the action (12) be independent of the choice of central term. This requirement will again be written as an algebraic identity involving the tensors $\eta_{1 \cdots n}$.

Let us then add a small central term $\kappa_{a b}$ to $\widehat{F}_{a b}$, and at the same time subtract the same $\kappa_{a b}$ from the effective metric $\Gamma_{a b}$. The two effects must 
compensate each other, yielding a vanishing total variation of the action. Adding $\kappa$ to $\widehat{F}$ means that

$$
-i\left[\lambda^{a}, \lambda^{b}\right] \rightarrow-i\left[\lambda^{a}, \lambda^{b}\right]+\Delta^{a b}
$$

with

$$
\Delta=-\theta \kappa \theta
$$

Therefore, a term with $n+2$ coordinate functions $\lambda^{a}$ will go into a term with $n$ functions $\lambda^{a}$. More precisely, the variation of a term

$$
\left(\lambda^{1} \star \cdots \star \lambda^{n+2}\right) \eta_{1 \cdots n+2}
$$

will be of the form

$$
-\left(\lambda^{1} \star \cdots \star \lambda^{n}\right)(\bar{\Delta} \eta)_{1 \cdots n}
$$

where $(\bar{\Delta} \eta)_{1 \cdots n}$ is again gauge invariant and depends on $\eta_{1 \cdots n+2}$ and on $\Delta^{a b}$. We will show in the appendix (Lemma 6) that

$$
(\bar{\Delta} \eta)_{1 \cdots n}=-\frac{i}{2} \Delta^{a b}\left(\eta_{1 \cdots n a b}+\eta_{1 \cdots a n b}+\cdots\right),
$$

where $\cdots$ indicates all the terms with the indices $1, \cdots, n$ in increasing order, and the two contracted indices $a, b$ in all possible positions with $a$ preceding $b$. Let us just note that, for $n=2$, the above result follows from (14), since a gauge invariant $\eta_{a b}$ is necessarily antisymmetric, and therefore $\lambda^{a} \star \lambda^{b} \eta_{a b}=\frac{1}{2}\left[\lambda^{a}, \lambda^{b}\right] \eta_{a b}$.

As noted previously, the variation (14) must be compensated by a corresponding change in the metric $\Gamma \rightarrow \Gamma-\kappa$. In particular, in expression (12) this will affect both the measure of integration and the tensors $\eta_{1 \cdots n}$. The measure changes by

$$
\operatorname{det}^{\frac{1}{2}} \Gamma \rightarrow \operatorname{det} \frac{1}{2} \Gamma\left(1+\frac{1}{2} \kappa_{a b} \Gamma^{a b}\right),
$$

and the tensors by

$$
\eta \rightarrow \eta-\kappa_{a b} \frac{\partial}{\partial \Gamma_{a b}} \eta
$$

Noting that $\kappa_{a b} \Gamma^{a b}=\gamma_{a b} \Delta^{a b}$ and that

$$
-\kappa_{a b} \frac{\partial}{\partial \Gamma_{a b}}=(\gamma \Delta \gamma)_{a b} \frac{\partial}{\partial \gamma_{a b}}
$$


we then conclude that the variation of a term $\left(\lambda^{1} \star \cdots \star \lambda^{n}\right) \eta_{1 \cdots n}$ will be

$$
\left(\lambda^{1} \star \cdots \star \lambda^{n}\right)(\bar{\delta} \eta)_{1 \cdots n}
$$

where

$$
\bar{\delta}=\frac{1}{2}\left(\gamma_{a b} \Delta^{a b}\right)+(\gamma \Delta \gamma)_{a b} \frac{\partial}{\partial \gamma_{a b}}
$$

Let us now combine the two variations. To this end, recall that the sum of terms in (12) runs only over even values of $n$. In particular we will say that a tensor $\eta_{1, \cdots, 2 L}$ with $2 L$ indices is of level $L$, and we will denote it with $\eta^{L}$. The operator $\bar{\Delta}$ lowers level by one, whereas $\bar{\delta}$ leaves the level invariant. In order to balance the two variations $\bar{\Delta}$ and $\bar{\delta}$ and to have invariance under a change of the central term we must then have that ${ }^{3}$

$$
\bar{\Delta} \eta^{L+1}=\bar{\delta} \eta^{L}
$$

\subsection{The Seiberg-Witten map following Jurco and Schupp}

In section 3.2 , we have written the action $S$ in terms of the functions $\lambda^{a}$, which implicitly depend on the non-commutative gauge potential $\widehat{A}=$ $\widehat{A}_{S W}(A, \theta)$. In order to analyze the independence of the action from the non-commutativity parameter $\theta$, it is convenient, as will become clear later, to rewrite $S$ in terms of the $\theta$-independent abelian potential $A$. To this end, we follow the analysis of Jurco and Schupp [8], whose work describes the Seiberg-Witten map $\widehat{A}_{S W}$ in an invariant way, which is best suited for our purposes. Most of this section is then nothing but a review of the ideas of [8], rewritten in the notation of this paper.

First recall that, on the manifold $M$, one can define, in a natural way, two distinct symplectic structures, defined by the two form $K$ and by the combination

$$
\omega=K+F,
$$

where $F=d A$ is the usual abelian field strength. Since $F$ is exact, the forms $K$ and $\omega$ define the same class in cohomology, and therefore, by Darboux's lemma; there is a diffeomorphism $\lambda: M \rightarrow M$ such that

$$
\lambda^{*} \omega=K \text {. }
$$

\footnotetext{
${ }^{3}$ We have checked invariance of the action under infinitesimal changes of $\Phi$ around $\Phi=-K$. On the other hand, this is sufficient, since invariance under variation of the central term is a property of the structure of the action, property which is independent of the specific value of $\Phi$.
} 
Starting from the two symplectic structures $K, \omega$, one can, first of all, define the corresponding Poisson brackets

$$
\{f, g\}_{K}=\theta^{a b} \partial_{a} f \partial_{b} g \quad\{f, g\}_{\omega}=\left(\omega^{-1}\right)^{a b} \partial_{a} f \partial_{b} g .
$$

It is clear that the two brackets are related by the diffeomorphism $\lambda$. More precisely, for any two functions $f, g$, one has the trivial identity

$$
\lambda^{*}\{f, g\}_{\alpha}=\left\{\lambda^{*} f, \lambda^{*} g\right\}_{\theta}
$$

where $\lambda^{*} f=f \circ \lambda$.

From the two symplectic structures $K$ and $\omega$ one can also construct, following Kontsevich [9], associated star-products

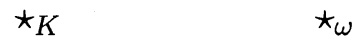

In particular, $\star_{K}$ is nothing but the usual Moyal product, since in the coordinates $x^{a}$ the symplectic structure $K$ is constant. The product $\star_{\omega}$ is, on the other hand, the full product of Kontsevich, which is expressed in terms of a complicated diagrammatic expression involving derivatives of the Poisson structure $\omega^{-1}$, and for which there is an elegant path-integral expression, by Cattaneo and Felder [7]. In what follows, we will not need the explicit form for $\star_{\omega}$. On the other hand, since $K$ and $\omega$ are related by diffeomorphism, it is a general result of Kontsevich that the two products $\star_{K}$ and $\star_{\omega}$ are equivalent. More precisely, there is a map $T$ defined on functions such that, for and functions $f$ and $g$,

$$
T\left(f \star_{\omega} g\right)=T f \star_{K} T g .
$$

The above expression is the analogue of expression (17), and in fact one can show that ${ }^{4}$

$$
T=\lambda^{*}(1+\cdots),
$$

where $\cdots$ are higher order terms (more precisely, if we replace $K, \omega \rightarrow$ $\frac{1}{\hbar} K, \frac{1}{\hbar} \omega$, then the terms in $\cdots$ are higher order in $\hbar$ ).

Given these facts, one can define, in terms of $T$, the Seiberg-Witten map as follows

$$
\lambda^{a}=T x^{a}=x^{a}+\theta^{a b} \widehat{A}_{b} .
$$

\footnotetext{
${ }^{4}$ We thank A. Cattaneo for pointing out that (19) is a simple consequence of formality, as defined in [9].
} 
We need to check that the map $\widehat{A}_{S W}$ implicitly defined above maps gauge orbits of the abelian theory to gauge orbits of the non-commutative theory. On one side, it is clear that different abelian potentials $A$ which are gaugeequivalent do give the same map $\widehat{A}$, since $T$ is only defined in terms of the combination $\omega$, which is itself gauge-invariant. Moreover, on the noncommutative side, we note that the map $T$ defined in (18) is only defined up to transformations

$$
T f \rightarrow \Lambda \star_{K} T f \star_{K} \Lambda^{-1},
$$

which leave (18) invariant ${ }^{5}$. This in turn generates gauge transformations

$$
\widehat{A}_{a} \rightarrow \widehat{A}_{a}+i \Lambda \star_{K} \partial_{a} \Lambda^{-1}+\Lambda \star_{K} \widehat{A}_{a} \star_{K} \Lambda^{-1},
$$

therefore showing that the transformation $\widehat{A}_{S W}$ does map gauge orbits into gauge orbits.

\subsection{Integration}

In the previous section we have reviewed the Jurco-Schupp construction of the Seiberg-Witten map. In this section we wish to discuss some issues about integration of functions over $M$ which are closely related to the discussion in the previous section, and which will be important in our subsequent discussion.

Corresponding to the two symplectic structures $K, \omega$, one has two volumeforms on $M$, respectively $d^{D} x$ det $\frac{1}{2} K$ and $d^{D} x \operatorname{det} \frac{1}{2} \omega$, which are related by the map $\lambda$. More specifically, if $f$ is a generic function which vanishes at infinity, using the fact that $\lambda^{*} \omega=K$, it is immediate to show that

$$
\int d^{D} x \operatorname{det}^{\frac{1}{2}} K \lambda^{*} f=\int d^{D} x \operatorname{det}^{\frac{1}{2}} \omega f .
$$

Similarly, we may consider, recalling from (19) that $T \sim \lambda^{*}$, the corresponding integral of $T f$. We then have, in general, that

$$
\int d^{D} x \operatorname{det}^{\frac{1}{2}} K T f=\int d^{D} x V(\omega) f,
$$

\footnotetext{
${ }^{5}$ The infinitesimal version of equation (20) is given by $T f \rightarrow T f+[\rho, T f]_{K}$. This change of $T$ is analogous to the fact that the map $\lambda$ is defined up to symplectomorphisms of the manifold $(M, K)$. In fact, if $\chi: M \rightarrow M$ is such that $\chi^{*} K=K$, then the composite map $\lambda \circ \chi$ still satisfies (16). Recalling that symplectomorphisms are generated by Hamiltonian flows, the change $\lambda^{*} \rightarrow(\lambda \circ \chi)^{*}$ is given infinitesimally by $\lambda^{*} f \rightarrow \lambda^{*} f+\left\{\rho, \lambda^{*} f\right\}_{K}$.
} 
where $V(\omega)$ is a volume element depending on $\omega$ and its derivatives, of the general form

$$
V(\omega)=\operatorname{det}^{\frac{1}{2}} \omega(1+\cdots),
$$

where $\cdots$ denotes, as in (19), higher order derivative corrections in $\omega^{-1}$ which vanish if $\omega$ is constant. Let me note that, since $\int d^{D} x \operatorname{det} \frac{1}{2} K f \star_{K} g=$ $\int d^{D} x \operatorname{det}^{\frac{1}{2}} K g \star_{K} f$, the ambiguity (20) in the definition of $T$ does not affect the definition (21) of $V(\omega)$, which really only depends on the symplectic structure $\omega$. Moreover, from the definition (18) of $T$, we have in general that

$$
\int d^{D} x V(\omega) f \star_{\omega} g=\int d^{D} x V(\omega) g \star_{\omega} f
$$

The explicit form of $V(\omega)$ is not know. On the other hand we will show in the rest of the paper that some properties of $V(\omega)$ can be proven indirectly, and this will suffice for our purposes.

\subsection{Back to the matrix action}

In this section we use the results just discussed on the Seiberg-Witten map and on integration to rewrite the action (12)

$$
\frac{1}{g_{s}} \sum_{n \text { even }} \int d^{D} x \operatorname{det}^{\frac{1}{2}} K\left(\lambda^{1} \star_{K} \cdots \star_{K} \lambda^{n}\right) \eta_{1 \cdots n}
$$

in an almost final form (we are now showing in the star-products $\star_{K}$ the explicit dependence on the symplectic structure). Using the facts that $\lambda^{a}=$ $T x^{a}$ and that $T\left(f \star_{\omega} g\right)=T f \star_{K} T g$, one quickly sees that

$$
\left(\lambda^{1} \star_{K} \cdots \star_{K} \lambda^{n}\right)=T\left(x^{1} \star_{\omega} \cdots \star_{\omega} x^{n}\right) .
$$

Using then equation (21) on integration one concludes that the action (12) can be rewritten as

$$
S=\frac{1}{g_{s}} \sum_{n \text { even }} \int d^{D} x V(\omega)\left(x^{1} \star_{\omega} \cdots \star_{\omega} x^{n}\right) \eta_{1 \cdots n} .
$$

The above action is written almost exclusively in terms of the closed string parameters $A, g+B$ and $g_{s}$. Moreover it is explicitly a gauge invariant function of $A$, since the dependence on the gauge potential is uniquely through the gauge invariant expression $\omega=K+F$. On the other hand, the action $S$ 
above still depends on the parameter $\theta$, through the definition of $\omega$ - which effects $\star_{\omega}$ and $V(\omega)$ - and through the effective metric $\gamma=g+B-K-$ which is the building block for the tensors $\eta$. However the action $S$ must be independent of $\theta$, and the analysis of this requirement will be the subject of the rest of the paper.

REMARK. We are now in a position to give some very intuitive arguments for the appearance of the full Kontsevich product in the effective action. The arguments which follow are vague and not precise. On the other hand, they provide a useful intuition, which, if made rigorous, could be of importance.

Let us start by recalling $[3,4]$ that the effective action can be considered as the partition function for an open string sigma model

$$
S(A) \propto \int D X e^{-I_{S}-I_{A}}
$$

where

$$
\begin{aligned}
I_{S} & =\frac{1}{\alpha^{\prime}} \int_{\Sigma} g_{a b} \partial X^{a} \bar{\partial} X^{b} \\
I_{A} & =\int_{\Sigma} B+\int_{\partial \Sigma} A
\end{aligned}
$$

If we consider the naive limit $\alpha^{\prime} \rightarrow 0$, the term $I_{S}$ dominates, and we should consider $I_{A}$ as a perturbation. On the other hand, we recall that, in [1], Seiberg and Witten consider the limit $\alpha^{\prime}, g_{a b} \rightarrow 0$, with $g_{a b} / \alpha^{\prime} \rightarrow 0$. Therefore, in this case, the dominating term is $I_{A}$. We also note that

$$
I_{A}=\int_{\Sigma}(B+F)
$$

and that the above is nothing but the Cattaneo-Felder model [7]

$$
\int \eta_{a} \wedge d X^{a}+\frac{1}{2} \alpha^{a b}(X) \eta_{a} \wedge \eta_{b}
$$

in the special case of invertible Poisson structure $\alpha^{a b}$, with $\alpha^{-1}=B+F$. In this case, one can integrate out the one-forms $\eta_{i}$, which appear quadratically, and recover (23). We recall that the perturbation theory of the Cattaneo-Felder model generates the Kontsevich graphs, which are the basis of the product $\star_{B+F}$. One then expects (in an undoubtedly vague way) to obtain effective actions based on the full Kontsevich product. Moreover one expects to obtain, among the various products considered in [9], the simplest one, defined using the harmonic angle map. 
Along the same lines one could also expand (22) in powers of $I_{S}$ and obtain an expansion like

$$
\int D X e^{-I_{A}} I_{S} I_{S} \sim \int d^{D} x V(\omega)\left[x^{a}, x^{b}\right]_{\omega} \star_{\omega}\left[x^{c}, x^{d}\right]_{\omega} g_{a c} g_{b d}
$$

where the RHS above is nothing but the $\widehat{F}^{2}$ term in the action, which dominates in the $\alpha^{\prime} \rightarrow 0$ limit of [1].

\subsection{Behavior at infinity and cyclic tensors}

We have seen that the general action describing the dynamics of gauge fields is of the form

$$
\frac{1}{g_{s}} \sum_{n \text { even }} \int d^{D} x V(\omega)\left(x^{1} \star_{\omega} \cdots \star_{\omega} x^{n}\right) \eta_{1 \cdots n}
$$

Let us now proceed by first concentrating on a single term in the sum (24). In particular let us focus on the expression

$$
\eta(x)=x^{1} \star_{\omega} \cdots \star_{\omega} x^{n} \eta_{1 \cdots n}
$$

The above clearly defined a function $\eta$ of the coordinates $x$, which is written in terms of $\omega^{-1}$ and its derivatives. We will assume throughout the paper that

$$
F(x) \rightarrow 0 \quad \text { when } x \rightarrow \infty
$$

This implies that, for large $x$, the symplectic structure $\omega \rightarrow K$ becomes constant, and that the star-product $\star_{\omega}$ becomes the Moyal product $\star_{K}$ with respect to $\theta$. In general then, for $x \rightarrow \infty$, the function $\eta(x)$ is a polynomial of degree $n$ in the coordinates $x^{a}$. If we assume further that $\eta_{1 \cdots n}$ is a gauge invariant tensor, then we can quickly see that, again for $x \rightarrow \infty$,

$$
\eta(x+\varepsilon)-\eta(x)=\varepsilon^{1} x^{2} \star_{K} \cdots \star_{K} x^{n}\left(\eta_{12 \cdots n}+\eta_{21 \cdots n}+\cdots\right)=0
$$

and therefore the function $\eta(x)$ approaches a constant $\eta_{\infty}$ at infinity. Similarly, the volume form $V(\omega)$ converges to the constant $\operatorname{det}^{\frac{1}{2}} K$ as $x \rightarrow \infty$. It is then clear that the integral $\int d^{D} x V(\omega) \eta$ in general diverges unless $\eta_{\infty}=0$. In order to define the action properly we should replace

$$
\int d^{D} x V(\omega) \eta \rightarrow \int d^{D} x V(\omega)\left[\eta-\eta_{\infty}\right]
$$

thereby eliminating the infinities coming from integration over an infinite world-volume. Let me note that, since $T 1=1$, one has $\int d^{D} x V(\omega)=$ 
$\int d^{D} x \operatorname{det}^{\frac{1}{2}} K$, and therefore the subtraction (25) is independent of $F$. Replacement (25) is then nothing but a constant addition to the action.

We will now show how the subtraction (25) can be achieved in an invariant way, without explicitly considering the behavior at infinity. First let us recall that, for functions $f$ and $g$ which vanish at infinity, we have that

$$
\int d^{D} x V(\omega) f \star_{\omega} g=\int d^{D} x V(\omega) g \star_{\omega} f
$$

We are therefore tempted to say that the integral $\int d^{D} x V(\omega)\left(x^{1} \star_{\omega} \cdots \star_{\omega} x^{n}\right)$ is invariant under cyclic permutations of the indices $1,2, \cdots, n$. This is, on the other hand, not quite correct, since the coordinate functions $x^{a}$ which enter in expression (24) clearly do not vanish for $x \rightarrow \infty$. Nonetheless let us, for the moment, blindly assume cyclicity of the integral. We may then substitute, in expression (24) for the action, the tensors $\eta_{1 \cdots n}$ with the cyclically symmetrized tensors

$$
\tau_{1 \cdots n}=\frac{1}{n}\left(\eta_{1 \cdots n}+\operatorname{cyc}_{1 \cdots n}\right),
$$

where $\operatorname{cyc}_{1 \cdots n}$ denotes the sum over cyclic permutations of the indices $1, \cdots, n$. Gauge invariance of the tensors $\eta$ then translates into the following algebraic property satisfied by the tensors $\tau$

$$
\tau_{123 \cdots n}+\tau_{213 \cdots n}+\tau_{231 \cdots n}+\cdots+\tau_{23 \cdots 1 n}=0 .
$$

Note that the above expression is very similar to (13), with the only difference that the moving index 1 runs only over the cyclically independent orderings, and therefore the last term in (13) is absent in (27). Tensors which satisfy the above relation will be called cyclic gauge invariant (CGI). We leave the proof of (27) to the appendix (Lemma 1).

We may now consider, similarly to the previous analysis, the function

$$
\tau(x)=x^{1} \star_{\omega} \cdots \star_{\omega} x^{n} \tau_{1 \cdots n}
$$

and in particular its behavior at infinity. As shown in the appendix (Lemma 2 ), for $n$ even (which is the case relevant to equation (24)) one has that

$$
\tau(x) \rightarrow 0
$$

for $x \rightarrow \infty$. Therefore the integral

$$
\int d^{D} x V(\omega) \tau
$$


is well defined. Moreover we will show in the appendix (Lemma 3) that, generically, one has that

$$
\int d^{D} x V(\omega) \tau=\int d^{D} x V(\omega)\left[\eta-\eta_{\infty}\right]
$$

so that we have lost nothing by assuming cyclicity ${ }^{6}$. In fact, using cyclic gauge invariant tensors, the subtraction which was needed in (25) in order to properly define the action $S(A)$ is automatically incorporated into the formalism. We will therefore consider, from now on, the final form of the action

$$
S=\frac{1}{g_{s}} \sum_{n \text { even }} \int d^{D} x V(\omega)\left(x^{1} \star_{\omega} \cdots \star_{\omega} x^{n}\right) \tau_{1 \cdots n}
$$

where the tensors $\tau$ are cyclic gauge invariant.

We have seen in section 3.3 that, in order for the tensors $\eta$ to define an action, they had to be gauge invariant and they had to satisfy $\bar{\Delta} \eta^{L+1}=$ $\bar{\delta} \eta^{L}$. These two properties impose restrictions on the cyclically symmetrized tensors $\tau$. Gauge invariance of the $\eta$ 's implies cyclic gauge invariance of the $\tau$ 's. The equation $\bar{\Delta} \eta^{L+1}=\bar{\delta} \eta^{L}$ implies a similar equation for the $\tau$ 's, which we now describe.

Consider a gauge invariant tensor $\eta_{1 \cdots n+2}$. We can construct, given $\Delta^{a b}$ and equation (15), the tensor $(\bar{\Delta} \eta)_{1 \cdots n}$, which is also gauge invariant for any choice of $\Delta^{a b}$. We may then consider the cyclically symmetric combination $g_{1 \cdots n}=\frac{1}{n}\left(\bar{\Delta} \eta_{1 \cdots n}+\right.$ cyc $\left._{1 \cdots n}\right)$, which will be a cyclic gauge invariant tensor. At first sight the tensor $g_{1 \cdots n}$ is a function of the original tensor $\eta_{1 \cdots n+2}$, but, as we will prove in the appendix (Lemma 7), it is actually just a function of the cyclically symmetrized tensor $\tau_{1 \cdots n+2}$. We will then denote the tensor $g_{1 \cdots n}$ with $(\bar{\Delta} \tau)_{1 \cdots n}$, where

$$
\begin{aligned}
(\bar{\Delta} \tau)_{1 \cdots n}= & -\frac{i}{2}\left(\frac{n+2}{n^{2}}\right) \Delta^{a b}\left[n \tau_{1 \cdots n a b}+(n-1) \tau_{1 \cdots a n b}+\cdots+0 \cdot \tau_{a 1 \cdots n b}\right] \\
& +\operatorname{cyc}_{1 \cdots n}
\end{aligned}
$$

Nothing on the other hand needs to be altered in the definition of the operator

$$
\bar{\delta}=\frac{1}{2}\left(\gamma_{a b} \Delta^{a b}\right)+(\gamma \Delta \gamma)_{a b} \frac{\partial}{\partial \gamma_{a b}}
$$

\footnotetext{
${ }^{6}$ Let me note that, although the functions $\tau$ and $\eta-\eta_{\infty}$ have the same integral, and therefore define the same functional of $A$, one has in general that $\tau \neq \eta-\eta_{\infty}$.
} 
which commutes with the symmetrization (26). We then have the requirement on the tensors $\tau$

$$
\bar{\Delta} \tau^{L+1}=\bar{\delta} \tau^{L}
$$

EXAMPLE. Let us compute, as an important example, the first non-vanishing tensor $\tau^{2}$. If we consider the expansion of the Born-Infeld action (with $\Omega=\widehat{F}-K$ )

$$
\sqrt{\operatorname{det}(\Gamma+\Omega)} \propto 1-\frac{1}{2} \Gamma^{a b} \Omega_{a b}+\frac{1}{4} \Gamma^{a c} \Gamma^{d b} \Omega_{a b} \Omega_{c d}+\frac{1}{8} \Gamma^{a b} \Omega_{a b} \Gamma^{c d} \Omega_{c d}
$$

we can quickly see that

$$
\begin{aligned}
\eta_{12}= & \frac{i}{2}\left(\gamma_{12}-\gamma_{21}\right) \\
\eta_{1234}= & -\frac{1}{4}\left(\gamma_{13} \gamma_{42}-\gamma_{23} \gamma_{41}+\gamma_{31} \gamma_{24}-\gamma_{32} \gamma_{14}\right) \\
& -\frac{1}{8}\left(\gamma_{12} \gamma_{34}-\gamma_{21} \gamma_{34}+\gamma_{12} \gamma_{43}-\gamma_{21} \gamma_{43}\right) .
\end{aligned}
$$

We may then compute the cyclically symmetrized tensors $\tau$. Clearly $\tau_{12}=0$. A simple computation also shows that

$$
\tau_{1234}=\frac{1}{4} g_{12} g_{34}+\frac{1}{4} g_{14} g_{23}-\frac{1}{2} g_{13} g_{24},
$$

where, we recall,

$$
g_{a b}=\frac{1}{2}\left(\gamma_{a b}+\gamma_{b a}\right)
$$

is the symmetric part of the tensor $\gamma_{a b}$. One can also check, given (30), that

$$
\bar{\Delta} \tau^{2}=0
$$

which is consistent with (29) and the fact that $\tau^{1}=0$.

\section{Operator Description}

In this section we leave momentarily the analysis of the effective action $S$, and we develop some formal tools which will allow us both to rewrite the various equations in a more compact and natural way, and also to tackle the problem of the independence of the action $S$ on the parameter $\theta$. 
First we analyze, within a general framework, the description of the action using operators. We then study a simple 2-dimensional example, which is not directly relevant to our more general situation, but which is completely tractable and which will be a useful frame of reference in discussing the general case. We then move on to the situation most relevant for this paper, and discuss, in that case, the generalization of the results obtained in the 2-dimensional setting.

\subsection{Operator representation of star products}

Let us consider first a flat symplectic structure $K$. We introduce a set of operators $J^{a}$ with commutation relations $\left[J^{a}, J^{b}\right]=i \theta^{a b}$, which can be represented on the Hilbert space $\mathcal{H}=L^{2}\left(\mathbb{R}^{D / 2}\right)$ as linear combinations of the standard $p, q$ operators in quantum mechanics (recall that we are assuming $\theta$ invertible). To any function $f$ on phase space $M=\mathbb{R}^{D}$ we can associate, using Weyl ordering, an operator $Q_{K}(f)$ acting on $\mathcal{H}$. It is then well known that, if $f$ and $g$ are two generic functions, then $Q_{K}(f) Q_{K}(g)=Q_{K}\left(f \star_{K} g\right)$. Moreover, if $f$ vanishes at infinity, one also has (up to an overall constant $(2 \pi)^{D / 2}$ which can be, for example, reabsorbed in the definition of the trace) that $\operatorname{Tr}\left(Q_{K}(f)\right)=\int d^{D} x \operatorname{det}^{\frac{1}{2}} K f$. We will call $Q$ a quantization map.

Consider now a general symplectic structure $\omega$. Since any two symplectic structures on $M$ are related by a diffeomorphism, one can follow section 3.4 and find a map $T$ on the space of functions such that, for general $f, g$, one has $T\left(f \star_{\omega} g\right)=T f \star_{K} T g$. One may then define a new quantization map $Q_{\omega}$, related to the symplectic structure $\omega$, by the following relation

$$
Q_{\omega}(f)=Q_{K}(T f) .
$$

It is then simple to show that

$$
Q_{\omega}(f) Q_{\omega}(g)=Q_{\omega}\left(f \star_{\omega} g\right)
$$

and that

$$
\left.\operatorname{Tr}\left(Q_{\omega} f\right)\right)=\int d^{D} x V(\omega) f
$$

Let us note that, for any fixed symplectic form $\omega$, the map $Q_{\omega}$ is actually only defined up to conjugation. Recall first that the map $T$ is defined up to a redefinition of the form $T f \rightarrow \widetilde{T} f=\Lambda \star_{K} T f \star_{K} \Lambda_{\widetilde{Q}}^{-1}$. Using $\widetilde{T}$ in equation (31), and letting $\chi=T^{-1} \Lambda$, we obtain a new map $\widetilde{Q}_{\omega}$ which reads, in terms 
of the original $Q_{\omega}$,

$$
\begin{aligned}
\widetilde{Q}_{\omega}(f) & =Q_{\omega}(\chi) Q_{\omega}(f) Q_{\omega}^{-1}(\chi) \\
& =Q_{\omega}\left(\chi \star_{\omega} f \star_{\omega} \chi^{-1}\right) .
\end{aligned}
$$

We now use this notation to rewrite the action $S$ in a compact and invariant way. First define the operators

$$
X^{a}=Q_{K}\left(\lambda^{a}\right)=Q_{\omega}\left(x^{a}\right) .
$$

Then the action (28) can then be compactly written as

$$
S=\frac{1}{g_{s}} \sum_{n \text { even }} \operatorname{Tr}\left(X^{1} \cdots X^{n}\right) \tau_{1 \cdots n} .
$$

The ambiguity (34) is reflected in a possible redefinition $X^{a} \rightarrow O X^{a} O^{-1}$, which on the other hand does not affect the action.

The action $S$ written above implicitly depends on a specific choice of non-commutativity parameter $\theta$. The dependence is two-fold. On one hand the tensors $\tau$ are built starting from the metric $\gamma$, which linearly depends on $K$. On the other hand, the parameter $K$ enters into the definition of the symplectic structure $\omega$, and therefore it implicitly determines the operators $X^{a}=Q_{\omega}\left(x^{a}\right)$. It is then clear that we need to understand the variation of the quantization map $Q_{\omega}$, when we add to $\omega_{a b}$ a constant antisymmetric matrix $\Delta_{a b}$. This is the subject of the next two sections. In particular, in the next section, we analyze this problem within a simple two-dimensional model, related to the general framework which we developed above. In this two-dimensional model the variation of $Q_{\omega}$ for $\omega \rightarrow \omega+\Delta$ can be completely analyzed. Moreover the solution will give us the correct ansatz to tackle the general problem.

\subsection{A simple 2-dimensional example}

The general framework of this section follows closely [13]. We consider the space $\mathcal{V}$ of complex functions on the complex plane $\mathbb{C}$, and the subspace $\mathcal{H} \subset \mathcal{V}$ of holomorphic functions. We then make $\mathcal{V}$ into a Hilbert space by choosing a real positive function $C$ on the complex plane and by letting the inner product of two function $\psi, \phi \in \mathcal{V}$ be given by

$$
\langle\psi \mid \phi\rangle=\int d^{2} z C \bar{\psi} \phi .
$$


Associated to $C$ we have a natural symplectic form $i \omega d z \wedge d \bar{z}$ on $\mathbb{C}$ with

$$
\omega=-\partial \bar{\partial} \ln C
$$

One may also consider the orthogonal projection

$$
\pi: \mathcal{V} \rightarrow \mathcal{H}
$$

which clearly depends on the choice of inner product on $\mathcal{V}$, and therefore on $C$. Given a generic function $f$, we may then consider the corresponding operator

$$
Q_{\omega}(f): \mathcal{H} \rightarrow \mathcal{H}
$$

defined by

$$
Q_{\omega}(f) \eta=\pi f \eta
$$

for $\eta \in \mathcal{H}$. In words, the operator $Q_{\omega}(f)$ first multiplies pointwise by $f-$ which is not assumed to be holomorphic - and then extracts the holomorphic part of the resulting function using the projector $\pi$. It is shown in [13] that, given two functions $f, g$,

$$
\begin{aligned}
Q_{\omega}(f) Q_{\omega}(g) & =Q_{\omega}\left(f \star_{\omega} g\right) \\
\operatorname{Tr}_{\mathcal{H}}\left(Q_{\omega}(f)\right) & =\int d^{2} z V(\omega) f
\end{aligned}
$$

where $\star_{\omega}$ is a holomorphic star product $\left(f \star_{\omega} g=f g\right.$ if either $\bar{f}$ or $g$ are holomorphic) related to $\omega$ and $V(\omega)=\omega(1+\cdots)$. The operator $Q_{\omega}$ actually depends on $C$, and not simply on $\omega$. On the other hand, a change of $C$ which leaves $\omega$ invariant changes the operators $Q_{\omega}(f)$ by conjugation, exactly as in (34).

We may now consider the variation $\omega \rightarrow \omega+\Delta$, with $\Delta$ an infinitesimal constant. This corresponds to

$$
C \rightarrow \widetilde{C}=C e^{-z \bar{z} \Delta} .
$$

We need to understand the change in $\pi$. Let then $\langle\|\rangle$ denote the original inner product with $C$, and let $|n\rangle$ be an orthonormal basis for $\mathcal{H}$. Then $\pi=\sum_{n}|n\rangle\langle n|$. It is easy to show that the vectors

$$
|\widetilde{n}\rangle=|n\rangle+\frac{\Delta}{2} \sum_{m}|m\rangle\langle m|z \bar{z}| n\rangle
$$


satisfy $\left\langle\widetilde{n}\left|e^{-z \bar{z} \Delta}\right| \widetilde{m}\right\rangle=\delta_{n, m}$ (to first order in $\Delta$ ) and that the new projection $\widetilde{\pi}$ related to $\widetilde{C}$ is given by $\widetilde{\pi}=\sum_{n}|\widetilde{n}\rangle\langle\widetilde{n}| e^{-z \bar{z} \Delta}$. Using the explicit expression for $|\widetilde{n}\rangle$, one can then show that

$$
\tilde{\pi}=\pi+\Delta(\pi z \bar{z} \pi-\pi z \bar{z}) .
$$

Let now $f$ be a generic function and $F=Q_{\omega}(f)=\pi f$. Noting that $\pi z=z$ one can show that

$$
\begin{aligned}
Q_{\omega+\Delta}(f) & =\tilde{\pi} f=F+\Delta(\pi \bar{z} z \pi f-\pi \bar{z} f z) \\
& =F+\Delta\left(\bar{Z} Z F-Q_{\omega}(\bar{z} f) Z\right)
\end{aligned}
$$

where $Z=Q_{\omega}(z)$ and $\bar{Z}=Q_{\omega}(\bar{z})$. Using the fact that the star product is holomorphic and that $Q_{\omega}(\bar{z} f)=Q_{\omega}\left(\bar{z} \star_{\omega} f\right)=\bar{Z} F$, we arrive at the result

$$
Q_{\omega+\Delta}(f)=F+\Delta(\bar{Z} Z F-\bar{Z} F Z) .
$$

The exact form of the above equation depends on the specific quantization model we chose to analyze. On the other hand the general lesson that should be drawn is that the variation $Q_{\omega+\Delta}(f)-Q_{\omega}(f)$ contains $F$ and two powers of the coordinate operators $X^{a}=Q_{\omega}\left(x^{a}\right)$, with some ordering. We will use this intuition in the next section to compute $Q_{\omega+\Delta}(f)$ in the setting of section 4.1 .

\subsection{The quantization map $Q_{\omega+\Delta}$ in the general case}

We have seen, from the previous example, that, if $f$ is a generic function, then the variation $Q_{\omega+\Delta}(f)-Q_{\omega}(f)$ is proportional to $\Delta_{a b}$ and to the product of

$$
F=Q_{\omega}(f)
$$

and of two coordinate operators $X^{a}=Q_{\omega}\left(x^{a}\right)$ in some specific ordering. More precisely, the operator $Q_{\omega+\Delta}(f)$ must be equal to $F+\frac{i}{4} \Delta_{a b}\left(a X^{a} X^{b} F+\right.$ $\left.b F X^{a} X^{b}+c X^{a} F X^{b}\right)$, for some choice of the coefficients $a, b, c$. It is shown in the appendix (Lemma 4) that, in the case in which the underlying starproduct is that of Kontsevich, the correct coefficients are $a=b=1$, and $c=-2$.

We have then the basic relation

$$
Q_{\omega+\Delta}(f)=F+\frac{i}{4} \Delta_{a b}\left(X^{a} X^{b} F+F X^{a} X^{b}-2 X^{a} F X^{b}\right) .
$$


The above can be alternatively rewritten as

$$
Q_{\omega+\Delta}(f)=Q_{\omega}(f+R f) .
$$

where

$$
R f=\frac{i}{4} \Delta_{a b}\left(x^{a} \star_{\omega} x^{b} \star_{\omega} f+f \star_{\omega} x^{a} \star_{\omega} x^{b}-2 x^{a} \star_{\omega} f \star_{\omega} x^{b}\right) .
$$

As a consequence of the above facts, we have the following two results. First of all combining (37) and (32) we obtain

$$
f \star_{\omega+\Delta} g=f \star_{\omega} g-\frac{i}{2} \Delta_{a b}\left[x^{a}, f\right]_{\omega} \star_{\omega}\left[x^{b}, g\right]_{\omega} .
$$

Also, taking the trace of (36) and using (33) we deduce that, for functions $f$ vanishing at infinity,

$$
\int d^{D} x V(\omega+\Delta) f=\int d^{D} x V(\omega)[f+R f] .
$$

REMARK . The fact that the variation of the star-product $\star_{\omega}$ under the change $\omega \rightarrow \omega+\Delta$ is given by an expression involving a quadratic combination (39) of the coordinate functions $x^{a}$ can also be understood intuitively using the CattaneoFelder model. As in the remark in section 3.6, the argument is very vague, but it would be very useful to make it rigorous.

The star product $f \star_{\omega} g$ is given by the disk expectation value $\langle f(X(0)) g(X(1))\rangle$, with weight $\int D X \exp \left(-\int_{\Sigma} \omega\right)$. Therefore, under the change $\omega \rightarrow \omega+\Delta$, one has that $f \star_{\omega+\Delta} g-f \star_{\omega} g$ is given by $-\int_{\Sigma}\langle\Delta f(X(0)) g(X(1))\rangle$. But $\Delta=\frac{1}{2} \Delta_{a b} d X^{a} \wedge d X^{b}$, thus giving a quadratic expression in the coordinate functions.

\section{Invariance of the Action}

\subsection{Invariance under a change in $\theta$ and the basic commutator}

We now have all the tools that we need to tackle our main problem. Let me first recall were we stand. The action is given by

$$
S=\frac{1}{g_{s}} \sum_{n \text { even }} \operatorname{Tr}\left(X^{1} \cdots X^{n}\right) \tau_{1 \cdots n}
$$


where $X^{a}=Q_{\omega}\left(x^{a}\right)$ and the tensors $\tau$ are cyclic gauge invariant tensors built from $\gamma$. The tensors $\tau$ satisfy the consistency condition $\bar{\delta} \tau^{L}=\bar{\Delta} \tau^{L+1}$, where $\tau^{L}$ denotes the tensor at level $L$, with $2 L$ indices.

The action $S$ depends on a specific choice of non-commutativity parameter $\theta$. The dependence is two-fold, through $\gamma=g+B-K$ and through $\omega=K+F$. We have argued in previous sections that the total dependence on $\theta$ should vanish, and therefore the two variations of the action under a change of $K$ should compensate each other and sum to zero. This clearly imposes additional restrictions on the possible forms of the tensors $\tau$, which we now analyze.

Let us start by considering an infinitesimal variation of $K$ given by

$$
K \rightarrow K+\Delta
$$

where $\Delta_{a b}$ is an arbitrary antisymmetric constant matrix. The metric $\gamma$ then changes as follows

$$
\gamma \rightarrow \gamma-\Delta
$$

therefore implying a change in the tensors $\tau$ given by

$$
\tau \rightarrow \tau-\delta \tau
$$

where $\delta$ is the differential operator defined by

$$
\delta=\Delta_{a b} \frac{\partial}{\partial \gamma_{a b}} .
$$

On the other hand, the symplectic structure $\omega$ changes by a constant term

$$
\omega \rightarrow \omega+\Delta .
$$

Then, as discussed in the previous section, the coordinate operators $X^{c}$ change as

$$
X^{c} \rightarrow X^{c}+\frac{i}{4} \Delta_{a b}\left(X^{a} X^{b} X^{c}+X^{c} X^{a} X^{b}-2 X^{a} X^{c} X^{b}\right) .
$$

We can now discuss the variation of the term $\operatorname{Tr}\left(X^{1} \cdots X^{n-2}\right) \tau_{1 \cdots n-2}$. It will consists of two parts, coming from the variation of the tensor $\tau$ and from the variation of the coordinate functions $X^{a}$. The first part is simply

$$
-\operatorname{Tr}\left(X^{1} \cdots X^{n-2}\right)(\delta \tau)_{1 \cdots n-2} .
$$


The second will involve the trace of $n$ coordinates, and will be of the general form

$$
\operatorname{Tr}\left(X^{1} \cdots X^{n}\right)(\Delta \tau)_{1 \cdots n},
$$

where the tensor $(\Delta \tau)_{1 \cdots n}$ is built from $\tau_{1 \cdots n-2}$ and from $\Delta_{a b}$. Applying equation (42) to the expression $\operatorname{Tr}\left(X^{1} \cdots X^{n-2}\right)$ and rearranging cyclically under the trace it is easy to show that

$$
(\Delta \tau)_{1 \cdots n}=\frac{i}{2}\left(\frac{n-2}{n}\right)\left(\Delta_{12} \tau_{345 \cdots n}-\Delta_{13} \tau_{245 \cdots n}\right)+\operatorname{cyc}_{1 \cdots n}
$$

First we note that the above tensor $\Delta \tau$ is cyclic gauge invariant for any choice of $\Delta_{a b}$. This fact is proved in the appendix (Lemma 5). Moreover, the fact that both $\tau$ and $\Delta \tau$ are cyclic gauge invariant is crucial in a more careful derivation of (43). In fact, the use of cyclic symmetry under the trace is formally correct, and does give the correct answer. On the other hand, one needs to check that the formal manipulations can be justified, since the coordinate functions do not vanish at infinity, and therefore one might forget important boundary terms. The detailed proof of equation (43) is again given in Lemma 5 .

We can then finally state the main algebraic equation which must be satisfied by the tensors $\tau$ in order for the action (41) to be invariant under changes of $\theta$. Again indicating with $L$ the level of a tensor $\tau_{1, \cdots, 2 L}$ with $2 L$ indices, we have the basic equation

$$
\Delta \tau^{L}=\delta \tau^{L+1}
$$

which must be considered together with the equation

$$
\bar{\Delta} \tau^{L+1}=\bar{\delta} \tau^{L}
$$

previously analyzed.

The above two equations involve the basic operators $\Delta, \delta, \bar{\Delta}, \bar{\delta}$, which in general depend on two distinct antisymmetric matrices $\Delta_{a b}$ and $\Delta^{a b}$. Without loss in generality we can assume that

$$
\Delta_{a c} \Delta^{c b}=\delta_{a}^{b}
$$

In this case one has simple relations between the operators $\delta, \bar{\delta}$ and $\Delta, \bar{\Delta}$, which we now describe. First we introduce the operator $N$ which simply counts the number of indices of a tensor, and which is defined by

$$
N \tau_{1 \cdots n}=n \cdot \tau_{1 \cdots n} .
$$


It is then easy to see that, since $\tau_{1 \cdots 2 L}$ is built from $L$ copies of $\gamma$, one has that

$$
2 \gamma_{a b} \frac{\partial}{\partial \gamma_{a b}}=N
$$

Using the above relation one then discovers quickly that

$$
[\delta, \bar{\delta}]=N-\frac{D}{2},
$$

where, we recall, $D$ is the dimension of space-time.

The relation between $\Delta$ and $\bar{\Delta}$ requires, on the other hand, a very long a quite technical analysis, which we leave to the appendix. Fortunately, though, the answer is very simple, and completely parallel to the above results. In fact, as shown in Lemma 8, one has that

$$
[\bar{\Delta}, \Delta]=N-\frac{D}{2} \text {. }
$$

We see that the structure of the seemingly different pairs of operators $\bar{\Delta}, \Delta$ and $\delta, \bar{\delta}$ is actually very similar and compatible, and we will use the above results heavily in the next section to solve the invariance equations for the simple case $D=2$.

Let us conclude this section by discussing the general form of the solution of equations (44) and (45). We introduce the concept of lowest level tensor, by which we mean a CGI tensor $\rho$ which satisfies

$$
\bar{\Delta} \rho=\delta \rho=0 .
$$

A general solution of (44) and (45) will then consist of a lowest level tensor $\tau^{P}$ at level $P$, together with tensors $\tau^{L}$ at higher levels $L>P$, which are required in order to obtain an invariant action. At each level $L, \tau^{L}$ is determined using (44) and (45) in terms of the tensors of lower level, up to an addition $\tau^{L} \rightarrow \tau^{L}+\rho$ of a lowest level tensor $\rho$. Let us suppose that we can, given a $\tau^{P}$ satisfying (46), construct, in a canonical way, a tower of tensors $\tau^{L}, L>P$ so that (44) and (45) hold. We will then call the full set $\left\{\tau^{L}\right\}_{L>P}$ the invariant block generated by $\tau^{P}$. The above discussion then shows that a solution of (44) and (45) is, in general, a linear combination of invariant blocks. We will see in the next section that, in the simple case $D=2$, we will indeed be able to construct canonically invariant blocks starting from generic lowest level tensors.

NOTE. Recall, from the example in section 3.7, that $\tau^{2}$ is given by equation (30) 
and satisfies $\bar{\Delta} \tau^{2}=0$. It is trivial to check that also $\delta \tau^{2}=0$ holds, since $\tau^{2}$ depends only on the symmetric part of $\gamma_{a b}$. Therefore $\tau^{2}$ is a lowest level state, as is natural to expect.

To conclude, let us comment on the question of uniqueness of the solution of the recursion equations (44) and (45). As we just discussed, we do not expect the solution to be unique, since general solutions are in one to one correspondence with lowest level tensors. An appropriate way of thinking about (44) and (45) is probably by analogy with general relativity. In that case, one is free to write actions of different type, subject only to the general principle of covariance under diffeomorphisms on the underlying space-time manifold. In a similar way, equations (44) and (45) imply that the action not only must be invariant under reparametrizations of the world-volume of the brane, but must also be invariant under a more general set of transformations, parametrized by changes in $\theta$. It would then be of great practical importance to have an explicitly covariant notation, for which invariance under (44) and (45) is manifest.

\subsection{Summary of the basic results}

We now summarize, in a compact but self-contained way, the results of this paper. Invariant actions are described by tensors $\tau^{L}$ for $L \geq 2$ such that

1. The tensor $\tau^{L}$ has $2 L$ indices and is built from a basic matrix $\gamma_{a b}$.

2. The tensors $\tau^{L}$ are cyclic gauge invariant - i.e. are cyclic tensors which satisfy the algebraic relation

$$
\tau_{123 \cdots n}+\tau_{213 \cdots n}+\tau_{231 \cdots n}+\cdots+\tau_{23 \cdots 1 n}=0 .
$$

3. For any choice of antisymmetric matrix $\Delta_{a b}$ (with $\Delta^{a b}$ indicating the inverse of $\Delta_{a b}$ ) the tensors $\tau^{L}$ satisfy the basic relation

$$
\begin{aligned}
\Delta \tau^{L} & =\delta \tau^{L+1} \\
\bar{\Delta} \tau^{L+1} & =\bar{\delta} \tau^{L}
\end{aligned}
$$

where the differential operators $\delta, \bar{\delta}$ are given by

$$
\begin{aligned}
\delta & =\gamma_{a b} \frac{\partial}{\partial \gamma_{a b}} \\
\bar{\delta} & =\frac{1}{2}\left(\gamma_{a b} \Delta^{a b}\right)+(\gamma \Delta \gamma)_{a b} \frac{\partial}{\partial \gamma_{a b}}
\end{aligned}
$$


and the algebraic operators $\Delta, \bar{\Delta}$ are given by

$$
(\Delta \tau)_{1 \cdots n}=\frac{i}{2}\left(\frac{n-2}{n}\right)\left(\Delta_{12} \tau_{345 \cdots n}-\Delta_{13} \tau_{245 \cdots n}\right)+\operatorname{cyc}_{1 \cdots n}
$$

and

$$
\begin{aligned}
(\bar{\Delta} \tau)_{1 \cdots n}= & -\frac{i}{2}\left(\frac{n+2}{n^{2}}\right) \Delta^{a b} . \\
& \cdot\left[n \tau_{1 \cdots n a b}+(n-1) \tau_{1 \cdots a n b}+\cdots+0 \cdot \tau_{a 1 \cdots n b}\right]+\operatorname{cyc}_{1 \cdots n}
\end{aligned}
$$

The operators $\Delta, \bar{\Delta}, \delta, \bar{\delta}$ satisfy the commutation relations

$$
[\bar{\Delta}, \Delta] \tau^{L}=[\delta, \bar{\delta}] \tau^{L}=\left(2 L-\frac{D}{2}\right) \tau^{L} .
$$

\section{A Solution in 2 Dimensions}

In this section we describe a general constructive solution of the invariance equations (44) and (45) in dimension $D=2$. This is clearly a toy model, since gauge fields have no propagating degrees of freedom in 2 dimensions. On the other hand the solution is still highly non-trivial, and it exhibits many of the features which are expected to be present in the general $D$ dimensional case.

\subsection{The general strategy}

It is convenient, in two dimensions, to use on $M$ complex coordinates

$$
z=x^{1}+i x^{2} \quad \bar{z}=x^{1}-i x^{2}
$$

with a hermitian metric

$$
g_{z \bar{z}}=g
$$

and $g_{z z}=g_{\overline{z z}}=0$. Similarly, the antisymmetric tensors $B_{a b}$ and $K_{a b}$ have a single independent component

$$
B_{z \bar{z}}=i B \quad K_{z \bar{z}}=i K
$$

The tensor $\gamma_{a b}$ is then given by a single complex number

$$
\begin{aligned}
\gamma_{z \bar{z}} & =g+i(B-K)=x+i y \\
\gamma_{\bar{z} z} . & =\overline{\gamma_{z \bar{z}}}=x-i y
\end{aligned}
$$


Finally we will use $Z, \bar{Z}$ for the operators which correspond to the coordinates $z, \bar{z}$ under the map $Q_{\omega}$.

We adopt, in this section, a notation which is not well suited for the general $D$-dimensional case treated in the remainder of the paper, but which is more economical in the present setting. Consider a general term of level $L$ in the action

$$
\tau_{1 \cdots 2 L} \operatorname{Tr}\left(X^{1} \cdots X^{2 L}\right)
$$

and let the various indices $1,2, \cdots, 2 L$ run over their possible values $z, \bar{z}$. We obtain a sum consisting of traces of monomials in $Z, \bar{Z}$, multiplied by polynomials of degree $L$ in $x, y$. In particular, the monomials under the trace satisfy the following two properties

1. They are constructed with $L$ coordinates $Z$ and $L$ coordinates $\bar{Z}$.

2. Monomials which differ only by a cyclic permutation of the coordinate operators $Z, \bar{Z}$ should be considered, as we recall from section 3.7 , as identical.

We call objects which satisfy (1) and (2) cyclic words of level $L$ - or simply words - and we will denote with $W_{L}$ the space of their linear combinations (for example, for $L=2$, the space $W_{2}$ is spanned by the two words $Z Z \overline{Z Z}$ and $Z \bar{Z} Z \bar{Z}$ ). Also we let $P_{L}$ be the space of polynomials in $x, y$ of degree $L$.

Among the possible cyclic words in $W_{L}$, we must consider the subspace

$$
G_{L} \subset W_{L}
$$

of cyclic gauge invariant combinations. Following once more the discussion of section 3.7, we define the space $G_{L}$ as follows. First introduce canonical creation and annihilation operators $a$ and $a^{\dagger}$, which satisfy $\left[a, a^{\dagger}\right]=1$, and let $O$ be the space of polynomials in $a, a^{\dagger}$. Consider then a map

$$
r: W_{L} \rightarrow O
$$

defined by taking a word $w$ in $W_{L}$ and by constructing the operator $r(w)$ by replacing $Z, \bar{Z}$ with $a, a^{\dagger}$, and then by summing over the possible cyclic permutations. For example, we associate to the word $w=\overline{Z Z} Z Z$ the operator $^{7}$

$$
r(w)=a^{\dagger} a^{\dagger} a a+a a^{\dagger} a^{\dagger} a+a a a^{\dagger} a^{\dagger}+a^{\dagger} a a a^{\dagger} .
$$

\footnotetext{
${ }^{7}$ Note that the sum over cyclic permutations is crucial in order to have a well-defined map $r$, since $W_{L}$ consists of words defined only up to cyclic permutation of the letters $Z, \bar{Z}$. In the above example, the same word $w$ can be equally represented by any of the permutations $w=\overline{Z Z} Z Z=\bar{Z} Z Z \bar{Z}=Z Z \overline{Z Z}=Z \overline{Z Z} Z$, but the operator $r(w)$ is independent of the choice of representative for $w$.
} 
We then have that

$$
G_{L}=\operatorname{ker} r
$$

This equation restates the fact (proven in Lemma 2) that, given a cyclic gauge invariant tensors $\tau_{1 \cdots 2 L}$, the function $\left(x^{1} \star_{\omega} \cdots \star_{\omega} x^{2 L}\right) \tau_{1 \cdots 2 L}$ vanishes whenever $\omega=K$ is constant.

The operators $\delta, \bar{\delta}, \Delta, \bar{\Delta}$ act naturally on the spaces $P_{L}$ and $G_{L}$. In particular the operators $\delta$ and $\bar{\delta}$ act on the spaces $P_{L}$ of polynomials

$$
\begin{array}{cll}
\delta: & P_{L} \rightarrow P_{L+1} \\
\bar{\delta}: & P_{L} \rightarrow P_{L-1}
\end{array}
$$

Choosing, without loss in generality, $\Delta_{z \bar{z}}=\Delta^{z \bar{z}}=i$, we have

$$
\begin{aligned}
\delta & =\frac{\partial}{\partial y} \\
\bar{\delta} & =-y+2 x y \frac{\partial}{\partial x}-\left(x^{2}-y^{2}\right) \frac{\partial}{\partial y}
\end{aligned}
$$

One can check explicitly that $[\delta, \bar{\delta}]=2 x \partial_{x}+2 y \partial_{y}-1=2 L-1$.

The operators $\Delta, \bar{\Delta}$ on the other hand act on the spaces $G^{L}$

$$
\begin{array}{ll}
\Delta: & G_{L} \rightarrow G_{L+1} \\
\bar{\Delta}: & G_{L} \rightarrow G_{L-1}
\end{array}
$$

We will be more explicit on the precise form of $\Delta$ and $\bar{\Delta}$ in the next subsection, but we know, from the general arguments of section 5.1 , that $[\bar{\Delta}, \Delta]=$ $2 L-1$.

With this notation in place we can now easily construct invariant actions. In particular, we will first show how to canonically construct, starting from a lowest level term, a complete set of terms which combine into an invariant block. A general invariant action is then given, following the discussion at the end of section 5.1, by linear combinations of invariant blocks.

Let us then first describe the form of a lowest level term. In general, given the above discussion, a generic term in the action at level $L$ will be of the form

$$
S_{L}=\sum_{i} p_{L}^{i} g_{L}^{i}
$$


where the $g_{L}^{i}$ are a basis for $G_{L}$ and the $p_{L}^{i}$ are polynomials in $P_{L}$. A term $S_{P}=\sum_{i} p_{P}^{i} g_{P}^{i}$ (we will reserve $L$ for a general level index, and $P$ for lowest level states) will be of lowest level if

$$
\delta S_{P}=0 \quad \bar{\Delta} S_{P}=0
$$

The first equation implies that the polynomials $p_{P}^{i}$ depend uniquely on $x$, and therefore that $p_{P}^{i}=c_{i} x^{P}$. Then

$$
S_{P}=x^{P} g_{P}
$$

where $g_{P}=\sum_{i} c_{i} g_{P}^{i}$ satisfies, using the second equation in (47),

$$
\bar{\Delta} g_{P}=0 .
$$

Let us then start with a lowest level term $S_{P}$ and construct a full invariant block $S$ of the form

$$
\begin{aligned}
S & =\sum_{L \geq P} S_{L} \\
S_{L} & =p_{L} g_{L} .
\end{aligned}
$$

The above is invariant if

$$
\begin{aligned}
\Delta S_{L} & =\delta S_{L+1} \\
\bar{\delta} S_{L} & =\bar{\Delta} S_{L+1}
\end{aligned}
$$

To solve the above constraints we construct the higher level $g_{L}$ 's using $\Delta$

$$
g_{L+1}=\Delta g_{L}
$$

We must then find polynomials $p_{L}$ which satisfy

$$
\delta p_{L+1}=p_{L}
$$

and such that

$$
\begin{aligned}
\bar{\Delta} g_{L+1} & =c_{L} g_{L} \\
\bar{\delta} p_{L} & =c_{L} p_{L+1}
\end{aligned}
$$

for some constant $c_{L}$. First we compute $\bar{\Delta} g_{L+1}$. Using the fact that $[\bar{\Delta}, \Delta]=$ $2 L-1$, and that $\bar{\Delta} g_{P}=0$, we obtain

$$
\begin{aligned}
\bar{\Delta} g_{L+1} & =\bar{\Delta} \Delta^{L+1-P} g_{P}=((2 L-1)+\cdots+(2 P-1)) g_{L} \\
& =c_{L} g_{L}
\end{aligned}
$$


with

$$
c_{L}=(L+P-1)(L-P+1) .
$$

Using equation (50)

$$
p_{L+1}=\frac{1}{(L+P-1)(L-P+1)} \bar{\delta} p_{L}
$$

to define higher level polynomials, we can check, using $[\delta, \bar{\delta}]=2 L-1$ and $\delta p_{P}=0$, that the remaining equation (49) is satisfied, and that we have indeed a solution to the invariance equations.

\subsection{An important example}

In this subsection we use the general construction described above and apply it to a specific important example. In particular we show again that the basic $F^{2}$ term is lowest level, and we construct part of the invariant block constructed from it. We recover in particular the Born-Infeld action, and we compute the first non-trivial derivative corrections at level 4 which must be present in order to make the full action invariant.

We start by analyzing the explicit form of the operators $\Delta, \bar{\Delta}$, recalling that $\Delta_{z \bar{z}}=\Delta^{z \bar{z}}=i$. We define for convenience the field strength $F$ as

$$
F=[Z, \bar{Z}] .
$$

Then equation (42) reads, in the present case,

$$
\begin{aligned}
\Delta Z & =\frac{1}{4}(Z F+F Z) \\
\Delta \bar{Z} & =\frac{1}{4}(\bar{Z} F+F \bar{Z}) .
\end{aligned}
$$

The above then defines the action of $\Delta$ on $G_{L}$, since $\Delta$ acts as a derivation on each coordinate forming the words in $G_{L}$. Similarly $\bar{\Delta}$ is defined by

$$
\bar{\Delta} F=1 \text {. }
$$

More precisely, given a word $w \in W_{L}$, we cyclically rearrange the coordinates in each word so as to obtain a gauge invariant (not cyclic gauge invariant) form, containing only commutators. We then apply $\bar{\Delta}$ on each fundamental commutator $F$ as a derivation.

Let us the consider the term

$$
S_{2}=p_{2} g_{2}
$$


with

$$
p_{2}=x^{2}=\frac{1}{2} F^{2} .
$$

Clearly $\delta p_{2}=0$. Moreover $\bar{\Delta} g_{2}=F=0$, since we recall that, as a word in $W_{1}$, the commutator $F$ is zero. Therefore $S_{2}$ is a lowest level state, and we can construct the corresponding invariant block.

First we do some computations explicitly. The polynomials $p_{3}$ and $p_{4}$ are given by

$$
\begin{aligned}
& p_{3}=\frac{1}{3} \bar{\delta} p_{2}=y x^{2} \\
& p_{4}=\frac{1}{8} \bar{\delta} p_{3}=-\frac{1}{8} x^{4}+\frac{1}{2} y^{2} x^{2} .
\end{aligned}
$$

We then compute $g_{3}$ and $g_{4}$. First, applying the operation $\Delta$ to $F$ we obtain

$$
\Delta F=\frac{1}{2}\left(F^{2}+Z F \bar{Z}-\bar{Z} F Z\right) .
$$

This means that (recall that the RHS below is a word in $W_{3}$, and that cyclic rearrangements are allowed)

$$
\begin{aligned}
g_{3} & =\Delta g_{2}=\frac{1}{2} F(\Delta F)+\frac{1}{2}(\Delta F) F=F(\Delta F) \\
& =\frac{1}{2} F^{3}+\frac{1}{2}[F Z, F \bar{Z}]=\frac{1}{2} F^{3}
\end{aligned}
$$

The computation of $g_{4}$ is just slightly more complex, and we leave it for the appendix (Lemma 9). The result is

$$
g_{4}=\Delta g_{3}=F^{4}+\frac{1}{4} F[D F, \bar{D} F]
$$

where

$$
D \cdots=[Z, \cdots] \quad \bar{D} \cdots=[\bar{Z}, \cdots] .
$$

We can now combine the polynomials and the gauge invariant words. To make contact with standard notation we write the action for $y=0\left(\gamma_{a b}\right.$ symmetric) and revert to more standard notation

$$
\begin{aligned}
F & \rightarrow i F_{z \bar{z}}=i F \\
x & \rightarrow g^{z \bar{z}}=\frac{1}{g} \\
D & \rightarrow i D
\end{aligned}
$$


which give the following $U(N)$ lagrangian

$$
-\frac{1}{2 g^{2}} \operatorname{Tr}\left(F^{2}\right)-\frac{1}{8 g^{4}} \operatorname{Tr}\left(F^{4}+\frac{i}{2} F[D F, \bar{D} F]\right)+\cdots .
$$

We see that the above action, written up to level 4, contains the first part of the Born-Infeld action (in 2-dimensions there is no ambiguity about ordering of the $F^{2 n}$ terms), but already at level 4 we have derivative corrections, which are required for the total invariance of the action ${ }^{8}$.

Let us now schematically consider the higher terms $g_{L}$. We wish to sketch how one can recover, within the $F^{2}$ invariant block, the complete Born-Infeld action (a much more detailed discussion on this point and related issues will appear in [16]). To this end, we first note that, in general,

$$
g_{L}=c_{L} F^{L}+\text { derivative terms. }
$$

We have that $c_{2}=c_{3}=1 / 2, c_{4}=1, \cdots$. We can use the basic commutator $[\bar{\Delta}, \Delta]$ and the fact that $\bar{\Delta} F^{L}=L F^{L-1}$ to compute all the $c_{L}$ 's. In fact, applying the basic equation $[\Delta, \Delta]=2 L-1$ to $g_{L}$ (recalling that $\Delta g_{L}=g_{L+1}$ and that $\bar{\Delta}$ does not decrease the number of derivatives) one obtains the recursion relation

$$
(L+1)\left(\frac{c_{L+1}}{c_{L}}\right)-L\left(\frac{c_{L}}{c_{L-1}}\right)=2 L-1
$$

which is solved by $c_{L}=(L-2) c_{L-1}$, or by

$$
c_{L}=\frac{1}{2}(L-2) !
$$

The $F^{2}$ invariant block then contains the sum $\sum_{L} c_{L} p_{L}(x, y) F^{L}$. Let us consider more closely the polynomials $p_{L}$. First of all, from the general relation (51) one can easily show that the polynomials $p_{L}$ vanish for $L$ odd if $y=0$. On the other hand, for even levels, one has that

$$
p_{2 L}(x, 0)=d_{2 L} x^{2 L} .
$$

\footnotetext{
${ }^{8}$ It has been shown in [6] that, at level 4 , the effective action can be written only as the $F^{4}$ term, with no derivative corrections. This result is not in contradiction with equation (53), since one can always allow for field redefinitions. In particular, consider, in general $D$ dimensions, the field redefinition $A_{a} \rightarrow A_{a}+c F_{a b} D_{c} F_{b c}$. This induces a change in the action at level 4 , coming from the $F^{2}$ term, of the form $F_{d a} D_{d}\left(F_{a b} D_{c} F_{b c}\right)=$ $\frac{1}{2} F_{a b}\left[D_{c} F_{b c}, D_{d} F_{a d}\right]$, which in two dimensions is proportional to $F[D F, \bar{D} F]$. Therefore, for an appropriate choice of $c$, one can remove the derivative term in (53), thus resolving the apparent contradiction with [6].
} 
Therefore, the relevant part of the action is given by (again substituting $x \rightarrow 1 / g$ and $F \rightarrow i F)$

$$
\sum_{L} c_{2 L} d_{2 L} \frac{(-)^{L}}{g^{2 L}} \operatorname{Tr}\left(F^{2 L}\right)
$$

To compute the coefficients $d_{2 L}$, let us first note that, since $\delta p_{2 L-1}=p_{2 L-2}$, one must have that $p_{2 L-1}=d_{2 L-2} x^{2 L-2} y+o\left(y^{3}\right)$. This implies, using (51), that $d_{2 L}=-\frac{1}{4 L(L-1)} d_{2 L-2}$, which is solved by (recall that $d_{2}=1$ )

$$
d_{2 L}=\left(-\frac{1}{4}\right)^{L-1} \frac{1}{L !(L-1) !} .
$$

Therefore, equation (54) gives the complete Born-Infeld action

$$
-\sum_{L}\left(\frac{1}{2}\right)^{2 L-1} \frac{(2 L-2) !}{L !(L-1) !} \frac{1}{g^{2 L}} \operatorname{Tr}\left(F^{2 L}\right)=\operatorname{Tr} \sqrt{1-\frac{1}{g^{2}} F^{2}} .
$$

\section{Discussion}

In this paper we have analyzed in detail the general structure of the nonabelian Born-Infeld action, together with the higher $\alpha^{\prime}$ derivative corrections. We have shown how the requirement of invariance of the action under a change of non-commutativity scale $\theta$ imposes severe restrictions on the possible terms which can appear. More specifically, we can construct invariant actions starting from invariant blocks, which are themselves obtained from a lowest level term (in a loose sense, a pure derivative term). Terms at higher level are then constructed so as to achieve invariance under a change in $\theta$. A general action is then a linear combination of invariant blocks, with coefficients which must be determined from a different computation. No argument in this paper assumes supersymmetry, and the results are therefore valid in bosonic open string theory, as well as in superstring theory. In particular, supersymmetry will impose restrictions on the allowed linear combinations of invariant blocks, possibly determining in part, or even completely, the effective action.

Let us now comment on interesting directions of possible future investigation.

- It is first of all important to explicitly solve the invariance equations $\Delta \tau^{L}=\delta \tau^{L+1}$ and $\bar{\Delta} \tau^{L+1}=\bar{\delta} \tau^{L}$ in the general $D$-dimensional 
case. Similarly to the 2-dimensional case discussed in the text, we should study the algebra of operators $\Delta, \delta, \bar{\Delta}, \bar{\delta}$ given by the relations $[\bar{\Delta}, \Delta]=[\delta, \bar{\delta}]=2 L-D / 2$. The algebra now depends on more parameters, since the underlying matrix $\Delta_{a b}$ now has $D(D-1) / 2$ components. It is important, in particular, to have a canonical construction of higher level terms, starting from the lowest level. This would in turn give a canonical definition of invariant block.

- It is important to understand how invariant blocks appear in the underlying boundary conformal field theory. In particular, the relation between the analysis of this paper, which is at the level of the effective action, and conformal field theory is of importance both conceptually and from a practical point of view.

- The results of this paper do not depend on supersymmetry. Understanding the additional constraints imposed by SUSY is an important task for the future. A first step in this direction is the following. Given an invariant action, we may use T-duality to describe the weakcoupling physics of D-branes. In particular, we expect, in a supersymmetric theory, to have minima of the effective action corresponding to holomorphic curves, surfaces, $\cdots$. Very possibly, a careful restatement of this fact in terms of invariant blocks will impose constraints which must be satisfied in a supersymmetric theory.

- Given the invariant description of the action (41) as an operator trace, it is very tempting to resum the full series in one specific invariant block. In fact, although the action is usually written by artificially choosing a parameter $\theta$ and then by writing the expression in terms of coordinate operators $X^{a}$, it is nonetheless true that the operator $O(\theta)=\sum_{L} X^{1} \cdots X^{2 L} \tau_{1 \cdots 2 L}$ has a trace $\operatorname{Tr}(O(\theta))$ which is $\theta$ independent. It is then tempting to conjecture ${ }^{9}$ that the various operators $O(\theta)$ not only have the same trace, but are related by a unitary transformation, and are then isospectral.

\section{Acknowledgments}

I would like to thank M. Douglas, A. Connes, A. Cattaneo and B. Shoikhet for useful discussions. Also I want to thank the Institute for Advanced Study and MIT, and in particular N. Seiberg and W. Taylor, for hospitality during the completion of this work. This research is supported by a European Post-doctoral Institute Fellowship.

\footnotetext{
${ }^{9}$ The spectral nature of actions has been very much stressed by A.Connes.
} 


\section{Appendix}

Definition 1. A tensor $\eta_{1 \cdots n}$ is called gauge invariant (GI) if

$$
\eta_{123 \cdots n}+\eta_{213 \cdots n}+\eta_{231 \cdots n}+\cdots+\eta_{23 \cdots 1 n}+\eta_{23 \cdots n 1}=0 .
$$

A tensor $\tau_{1 \ldots n}$ is called cyclic gauge invariant (CGI) if

$$
\tau_{123 \cdots n}+\tau_{213 \cdots n}+\tau_{231 \cdots n}+\cdots+\tau_{23 \cdots 1 n}=0 .
$$

Lemma 1. (Section 3.7) Let $\eta_{1 \cdots n}$ be gauge invariant. Then

$$
\tau_{1 \cdots n}=\frac{1}{n}\left(\eta_{1 \cdots n}+\operatorname{cyc}_{1 \cdots n}\right)
$$

is cyclic gauge invariant.

PROOF. Let us write the left hand side of equation (55) in terms of $\eta$. Neglecting the multiplicative factor of $1 / n$, we have the expression

$$
\begin{aligned}
& \eta_{123 \cdots n}+\eta_{213 \cdots n}+\eta_{231 \cdots n}+\cdots+\eta_{23 \cdots 1 n}+ \\
& \eta_{23 \cdots n 1}+\eta_{13 \cdots n 2}+\eta_{31 \cdots n 2}+\cdots+\eta_{3 \cdots 1 n 2}+ \\
& \eta_{3 \cdots n 12}+\eta_{3 \cdots n 21}+\cdots \\
& \cdots \\
& \cdots+\eta_{1 n 23 \cdots n-1}+ \\
& \eta_{n 123 \cdots n-1}+\eta_{n 213 \cdots n-1}+\eta_{n 231 \cdots n-1}+\cdots+\eta_{n 23 \cdots n-1,1}
\end{aligned}
$$

The above expression contains $(n-1) n$ terms ( $n$ lines with $n-1$ terms each). Consider the sequence of terms in the order written, and assemble them now into groups of $n$ terms. It is then easy to see that each individual group vanishes since $\eta$ is gauge invariant.

Lemma 2. (Section 3.7) Let $\tau_{1 \cdots n}$ be cyclic gauge invariant and let

$$
\tau(x)=x^{1} \star_{\omega} \cdots \star_{\omega} x^{n} \tau_{1 \cdots n} .
$$

Then, for $x \rightarrow \infty, \tau(x)$ grows linearly with $x$. Moreover, if $n$ is even, then

$$
\tau(x) \rightarrow 0
$$

for $x \rightarrow \infty$.

PROOF. We use the fact that, at infinity, $\omega \rightarrow K$ approaches a constant, and that we can therefore compute the variation

$$
\begin{aligned}
\tau(x+\varepsilon)-\tau(x) & =\varepsilon^{1} x^{2} \star_{K} \cdots \star_{K} x^{n}\left(\tau_{12 \cdots n}+\cdots+\tau_{2 \cdots n 1}\right) \\
& =h(x),
\end{aligned}
$$


where

$$
h(x)=x^{2} \star_{K} \cdots \star_{K} x^{n} \varepsilon^{1} \tau_{12 \cdots n} .
$$

It is immediate to see that, for any $\varepsilon$, the tensor $\varepsilon^{1} \tau_{12 \cdots n}$ is gauge invariant in the indices $2, \cdots, n$ and that, using the results of section 3.7, the function $h(x)$ is constant at infinity. This implies that $\tau(x)$ is at most a linear function of the coordinates $x$, when $x \rightarrow \infty$. We now note that $x^{1} \star_{K} \cdots \star_{K} x^{n}$ is a polynomial of degree $n$, with monomials of degrees $n, n-2, n-4, \cdots$. In particular, if $n$ is even, we do not have a linear term and the function $\tau$ approaches a constant at infinity. We now wish to show that the constant is 0 . Consider the polynomial $x^{1} \star_{K} \cdots \star_{K} x^{n}$. It will be of the form

$$
x^{1} \star_{K} \cdots \star_{K} x^{n}=C^{1 \cdots n}+o(x) .
$$

We have just seen that we do not need to consider the $o(x)$ part, and we therefore just need to prove that $C^{1 \cdots n} \tau_{1 \cdots n}=0$. It is not difficult to show, using the Moyal product $\star_{K}$, that

$$
C^{1 \cdots n} \propto \sum_{\sigma}(-)^{J(\sigma)} \theta^{\sigma_{1} \sigma_{2}} \cdots \theta^{\sigma_{n-1} \sigma_{n}}
$$

where $J(\sigma)$ counts the number of pairs $\sigma_{2 i-1}, \sigma_{2 i}$ for which $\sigma_{2 i-1}>\sigma_{2 i}$. Then, in order to finish the proof, one has to show that the quantity

$$
\sum_{\sigma}(-)^{J\left(\sigma^{-1}\right)} \tau_{\sigma_{1} \cdots \sigma_{n}}
$$

vanishes for cyclic $\tau$ 's. Let us then fix a given permutation $\sigma$, and let me denote with $\pi$ the basic cyclic permutation $(1, \cdots, n) \rightarrow(2, \cdots, n, 1)$. Consider then the permutations $\rho_{k}=\pi^{k} \circ \sigma$. Let us first show that $(-)^{J\left(\rho_{k}^{-1}\right)}$ is alternating with $k$. In fact, for $k \rightarrow k+1$, almost all the pairs $\sigma_{2 i-1}, \sigma_{2 i}$ go into the pairs $\sigma_{2 i-1}+1, \sigma_{2 i}+1$. This is, on the other hand, not true for the single pair with either $\sigma_{2 i-1}$ or $\sigma_{2 i}$ equal to $n$, since $n \rightarrow 1$. Only this one pair changes the ordering of its components, and therefore the sign $(-)^{J\left(\rho_{k}^{-1}\right)}$ changes if $k \rightarrow k+1$. Consider then the set of permutations $\rho_{k}$, cyclic permutations of $\sigma$, for $0 \leq k<n$. This gives, in the sum (57),

$$
\pm\left(\tau_{\sigma_{1} \sigma_{2} \sigma_{3} \cdots \sigma_{n}}-\tau_{\sigma_{2} \sigma_{3} \cdots \sigma_{n} \sigma_{1}}+\tau_{\sigma_{3} \cdots \sigma_{n} \sigma_{1} \sigma_{2}}-\cdots-\tau_{\sigma_{n} \sigma_{1} \sigma_{2} \cdots \sigma_{n-1}}\right)
$$

The terms come with alternating signs, and since $n$ is even the number of + signs is equal to that of - signs. Moreover, all the terms are actually the same, since $\tau$ is cyclically symmetric. The above sum then vanishes. Partitioning the set of all permutations $\sigma$ in sets of cyclically related permutations, we can then show that the full sum (57) vanishes. 
Remark 1. (Lemmata 3 and 5) We make a general comment on integration of commutators, which will be useful in the rest of the appendix. Consider two functions $f$ and $g$, and look at the integral

$$
\int d^{D} x V(\omega)\left(f \star_{\omega} g-g \star_{\omega} f\right) .
$$

It $f$ and $g$ vanish at infinity, then the above integral vanishes, as was discussed in the main text. If, on the other hand, $f$ and $g$ do not go to zero for $x \rightarrow \infty$, we can proceed as follows. Assume that $\omega=K$ outside of a compact domain $D \subset M$ and consider the integral of $[f, g]_{\omega}$ over $D$

$$
I=\int_{D} d^{D} x V(\omega)[f, g]_{\omega}
$$

If $f, g=0$ on $\partial D$ and outside of $D$, the above expression vanishes, and therefore, in general, the integral (58) must reduce to a boundary integral over $\partial D$. We can then continuously deform $\omega \rightarrow K$ in the interior of $D$ without changing the integral. This means, in particular, that, for any functions $f$ and ,

$$
I=\int_{D} d^{D} x \operatorname{det} \frac{1}{2} K[f, g]_{K} .
$$

From the above arguments it is also clear that the above equality holds even if $f$ and $g$ depend themselves in a local way on $\omega$. For example, if $f=f_{1} \star_{\omega} f_{2}$, then we have

$$
I=\int_{D} d^{D} x \operatorname{det} \frac{1}{2} K\left[f_{1} \star_{K} f_{2}, g\right]_{K} .
$$

In practice, when integrating commutators, we can replace $\omega$ with $K$ in all the expressions without changing the integral.

Lemma 3. (Section 3.7) Let $\eta_{1 \cdots n}$ be gauge invariant and let

$$
\tau_{1 \cdots n}=\frac{1}{n}\left(\eta_{1 \cdots n}+\mathrm{cyc}_{1 \cdots n}\right) .
$$

Define

$$
\begin{aligned}
& \eta(x)=x^{1} \star_{\omega} \cdots \star_{\omega} x^{n} \eta_{1 \cdots n} \\
& \tau(x)=x^{1} \star_{\omega} \cdots \star_{\omega} x^{n} \tau_{1 \cdots n}
\end{aligned}
$$

with $\eta(x) \rightarrow \eta_{\infty}$ for $x \rightarrow \infty$. If $n$ is even, then

$$
\int d^{D} x V(\omega) \tau=\int d^{D} x V(\omega)\left[\eta-\eta_{\infty}\right]
$$


PROOF. First it is clear that

$$
\begin{aligned}
\tau(x)= & \frac{1}{n} \eta_{1 \cdots n}\left(x^{1} \star_{\omega} \cdots \star_{\omega} x^{n}+\right. \\
& +x^{2} \star_{\omega} \cdots \star_{\omega} x^{n} \star_{\omega} x^{1}+\cdots \\
& \left.+x^{n} \star_{\omega} x^{1} \star_{\omega} \cdots \star_{\omega} x^{n-1}\right) .
\end{aligned}
$$

Therefore, the difference $\eta-\tau$ is given by

$$
\begin{aligned}
\eta-\tau= & \frac{1}{n} \eta_{1 \cdots n}\left(\left[x^{1}, x^{2} \star_{\omega} \cdots \star_{\omega} x^{n}\right]_{\omega}\right. \\
& +\left[x^{1} \star_{\omega} x^{2}, x^{3} \star_{\omega} \cdots \star_{\omega} x^{n}\right]_{\omega}+\cdots \\
& \left.+\left[x^{1} \star_{\omega} \cdots \star_{\omega} x^{n-1}, x^{n}\right]_{\omega}\right) .
\end{aligned}
$$

Recalling remark 1, we will be done once we show that the RHS above is equal to

$$
\eta_{\infty}=\eta_{1 \cdots n} x^{1} \star_{K} \cdots \star_{K} x^{n}
$$

when we replace $\omega \rightarrow K$. We must therefore prove that

$$
\eta_{\infty}=\frac{1}{n} \eta_{1 \cdots n}\left(\left[x^{1}, x^{2} \star_{K} \cdots \star_{K} x^{n}\right]_{K}+\cdots+\left[x^{1} \star_{K} \cdots \star_{K} x^{n-1}, x^{n}\right]_{K}\right) .
$$

Both the LHS and the RHS above are constant, since $\eta$ is GI. Let us introduce a compact notation

$$
\begin{aligned}
\eta_{1 \cdots n} x^{1} \star_{K} \cdots \star_{K} x^{n} & \rightarrow[1 \cdots n] \\
\eta_{1 \cdots n} x^{2} \star_{K} \cdots \star_{K} x^{n} \star_{K} x^{1} & \rightarrow[2 \cdots n 1]
\end{aligned}
$$

Using formula (56) and the arguments which follow it, we can show that

$$
[k \cdots n 1 \cdots k-1]=(-1)^{k-1}[1 \cdots n]=(-1)^{k-1} \eta_{\infty} .
$$

Then the RHS of (59) is given by

$$
\begin{aligned}
& \frac{n-1}{n}[12 \cdots n]-\frac{1}{n}[23 \cdots n 1]-\cdots-\frac{1}{n}[n 1 \cdots n-1] \\
= & {[12 \cdots n]\left(\frac{n-1}{n}+\frac{1}{n}-\frac{1}{n}+\cdots+\frac{1}{n}\right)=} \\
= & {[12 \cdots n] }
\end{aligned}
$$

as was to be shown. 
Lemma 4. (Section 4.3) Let $f$ be a generic function, and let $F=Q_{\omega}(f)$, where $\omega$ is an arbitrary symplectic structure. Let also $\Delta_{a b}$ be a constant antisymmetric matrix. Then, to first order in $\Delta$,

$$
Q_{\omega+\Delta}=F+\frac{i}{4} \Delta_{a b}\left(X^{a} X^{b} F+F X^{a} X^{b}-2 X^{a} F X^{b}\right) .
$$

PROOF. We start by noting that, if $\omega=K$ is constant, a simple computation using the Moyal product $\star_{K}$ shows that

$$
\begin{aligned}
f \star_{K+\Delta} g-f \star_{K} g & =-\frac{i}{2}(\theta \Delta \theta)^{a b} \partial_{a} f \star_{K} \partial_{b} g= \\
& =-\frac{i}{2} \Delta_{a b}\left[x^{a}, f\right]_{K} \star_{K}\left[x^{b}, g\right]_{K} .
\end{aligned}
$$

We must then consider the product $f \star_{\omega+\Delta} g$ for general $\omega$. As always, we look for a map $T$ such that $T\left(f \star_{\omega+\Delta} g\right)=T f \star_{\omega} T g$. If we work to first order in $\Delta$, and accordingly let $T=1+R$ (with $R$ or order $\Delta$ ), one has that

$$
f \star_{\omega+\Delta} g-f \star_{\omega} g=R f \star_{\omega} g+f \star_{\omega} R g-R\left(f \star_{\omega} g\right) .
$$

The map $T=1+R$ also relates $Q_{\omega+\Delta}$ and $Q_{\omega}$ as follows

$$
Q_{\omega+\Delta}(f)=Q_{\omega}(f+R f) .
$$

We have seen from the example in section 4.2 that the we should consider general variations $Q_{\omega+\Delta}(f)-Q_{\omega}(f)$ of the form

$$
\frac{i}{4} \Delta_{a b}\left(a X^{a} X^{b} F+b F X^{a} X^{b}+c X^{a} F X^{b}\right),
$$

where $a, b, c$ are constants which we must determine. It is then clear that

$$
R f=\frac{i}{4} \Delta_{a b}\left(a x^{a} \star x^{b} \star f+b f \star x^{a} \star x^{b}+c x^{a} \star f \star x^{b}\right) .
$$

Using the above fact in the RHS of equation (61), and comparing, for $\omega=K$, with the RHS of equation (60), we obtain that $a=b=1, b=-2$, as was required.

Lemma 5. (Section 5.1) Let $\tau_{1 \cdots n-2}$ be a cyclic gauge invariant tensor, and let $\Delta_{a b}$ be a constant antisymmetric matrix. Then the tensor

$$
(\Delta \tau)_{1 \cdots n}=\frac{i}{2}\left(\frac{n-2}{n}\right)\left(\Delta_{12} \tau_{345 \cdots n}-\Delta_{13} \tau_{245 \cdots n}\right)+\operatorname{cyc}_{1 \cdots n}
$$

is itself cyclic gauge invariant. Moreover, if $\omega$ is a generic symplectic structure and $X^{a}=Q_{\omega}\left(x^{a}\right)$, then, under the variation $\omega \rightarrow \omega+\Delta$, the operator $\operatorname{Tr}\left(X^{1} \cdots X^{n-2}\right) \tau_{1 \cdots n-2}$ varies by $\operatorname{Tr}\left(X^{1} \cdots X^{n}\right)(\Delta \tau)_{1 \cdots n}$ whenever $n$ is even. 
PROOF. First we show that the tensor $\Delta \tau$ is indeed cyclic gauge invariant. Written in full, we want to show that the following sum

$$
\begin{aligned}
& \left(\Delta_{12} \tau_{345 \cdots n}-\Delta_{13} \tau_{245 \cdots n}\right)+\operatorname{cyc}_{123 \cdots n} \\
& \left(\Delta_{21} \tau_{345 \cdots n}-\Delta_{23} \tau_{145 \cdots n}\right)+\operatorname{cyc}_{213 \cdots n} \\
& \cdots \\
& \left(\Delta_{23} \tau_{45 \cdots 1 n}-\Delta_{24} \tau_{35 \cdots 1 n}\right)+\operatorname{cyc}_{23 \cdots 1 n}
\end{aligned}
$$

vanishes. To this end, we consider three significant cases, with the hope that the reader can understand from them the general line of the argument.

Let us first consider, within the above sum, terms which are proportional to $\Delta_{12}$. They come only from the first and the last line and are

$$
\tau_{345 \cdots n}-\tau_{n 34 \cdots n-1}=0 .
$$

Similarly, terms proportional to $\Delta_{21}$ come from the second and third line and exactly cancel each other. Consider now terms proportional, say, to $\Delta_{23}$. These terms are present in every line of the above sum, and they are

$$
\left(\tau_{145 \cdots n}-\tau_{145 \cdots n}\right)+\left(\tau_{145 \cdots n}+\tau_{415 \cdots n}+\cdots+\tau_{45 \cdots 1 n}\right)
$$

The above vanishes since $\tau$ is cyclic gauge invariant. Finally we consider terms which are proportional to $\Delta_{2 n}$. In this final case, we can check that no terms in the sum (62) contain $\Delta_{2 n}$. The reader can convince him or herself that all other combination of indices fall in one of these three cases.

Remark 2. Let us note that, in the above proof, we have not used the fact that $\Delta_{a b}$ is antisymmetric. In fact, we have shown more generally that the tensor

$$
\left(A_{12} \tau_{345 \cdots n}-A_{13} \tau_{245 \cdots n}\right)+\operatorname{cyc}_{1 \cdots n}
$$

is cyclic gauge invariant for any choice of $A$, whenever $\tau$ itself is CGI.

We now move to the second part of the lemma. Introduce the following two functions on $M$

$$
\begin{aligned}
& A(x)=x^{1} \star_{\omega} \cdots \star_{\omega} x^{n-2} \tau_{1 \cdots n-2} \\
& B(x)=x^{1} \star_{\omega} \cdots \star_{\omega} x^{n}(\Delta \tau)_{1 \cdots n} .
\end{aligned}
$$

Since both $\tau$ and $\Delta \tau$ are CGI, the two functions $A$ and $B$ tend to 0 as $x \rightarrow \infty$ (we are assuming $n$ even). Recall that, under a variation $\omega \rightarrow \omega+\Delta$, the star product of two functions $f \star_{\omega} g$ changes by $R f \star g+f \star R g-R(f \star g)$ (we 
do not show the explicit $\omega$ dependence in $\star$ ), where $R$ is given in equation (38). Therefore, given three functions, the variation of $f \star g \star h$ is $R f \star g \star$ $h+f \star R g \star h+f \star g \star R h-R(f \star g \star h)$, and similarly for products of more functions. In particular, the variation of the function $A$ is given by

$$
A \rightarrow A+C-R A
$$

where

$$
C(x)=R x^{1} \star_{\omega} \cdots \star_{\omega} x^{n-2} \tau_{1 \cdots n-2}+\cdots+x^{1} \star_{\omega} \cdots \star_{\omega} R x^{n-2} \tau_{1 \cdots n-2} .
$$

Since we are interested in traces of operators, we must consider also the variation coming from the change of integration measure. We use equations (40) and (63), together with the fact that $A$ vanishes at infinity, to show that the variation of

$$
\operatorname{Tr}\left(X^{1} \cdots X^{n-2}\right) \tau_{1 \cdots n-2}=\int d^{D} x V(\omega) A
$$

is simply given by

$$
\int d^{D} x V(\omega) C
$$

We must then prove that

$$
\int d^{D} x V(\omega)(B-C)=0 .
$$

It is simple to show that the function $B$ is obtained by cyclically rearranging the coordinate functions which build $C$. Following remark 1 , the integral above reduces to a boundary term, and to show that it vanishes we just need to prove that $B=C$ whenever $\omega=K$ is constant. On one side, we know that $B=0$ for $\omega=K$, since $\Delta \tau$ is cyclic gauge invariant. We then need to prove that, for constant symplectic structures, $C=0$. This is shown in two steps. First look at the operation $R$ on coordinate functions in the case of flat symplectic structure

$$
\begin{aligned}
R x^{a} & =\frac{i}{4} \Delta_{b c}\left(x^{b} \star_{K} x^{c} \star_{K} x^{a}+x^{a} \star_{K} x^{b} \star_{K} x^{c}-2 x^{b} \star_{K} x^{a} \star_{K} x^{c}\right) \\
& =M_{b}^{a} x^{b}
\end{aligned}
$$

with

$$
M_{b}^{a}=-\frac{1}{2} \theta^{a c} \Delta_{c b}
$$


It is then clear that

$$
\begin{aligned}
C(x) & =x^{1} \star_{\omega} \cdots \star_{\omega} x^{n-2} \pi_{1 \cdots n-2} \\
\pi_{1 \cdots n-2} & =M_{1}^{a} \tau_{a 2 \cdots n-2}+\cdots+M_{n-2}^{a} \tau_{1 \cdots n-3, a}
\end{aligned}
$$

It is now quite easy to show that $\pi$ is cyclic gauge invariant, therefore implying that $C=0$.

Lemma 6. (Section 3.3) Let $\eta_{1 \cdots n+2}$ be gauge invariant, and let us consider the combination $C=\left(\lambda^{1} \star \cdots \star \lambda^{n+2}\right) \eta_{1 \cdots n+2}$. If we add a central term $\Delta^{a b}$ to the commutator $-i\left[\lambda^{a}, \lambda^{b}\right]$, then the expression $C$ varies by $-\left(\lambda^{1} \star \cdots \star \lambda^{n}\right)(\bar{\Delta} \eta)_{1 \cdots n}$, where

$$
(\bar{\Delta} \eta)^{1 \cdots n}=-\frac{i}{2} \Delta^{a b}\left(\eta_{1 \cdots n a b}+\eta_{1 \cdots a n b}+\cdots\right)
$$

Moreover, for any $\Delta^{a b}$, the tensor $\bar{\Delta} \eta$ is gauge invariant.

PROOF. First it is clear that, if $\eta_{1 \cdots n}$ and $\nu_{1 \cdots m}$ are gauge invariant, then so is $(\eta \nu)_{1 \cdots n+m}=\eta_{1 \cdots n} \nu_{n+1 \cdots n+m}$. Moreover, if $d_{a}$ is any one-indexed tensor, then $(d \eta)_{1 \cdots n+1}=d_{1} \eta_{2 \cdots n+1}-\eta_{1 \cdots n} d_{n+1}$ is again gauge invariant. These two facts can either be checked algebraically, or one can simply note that they correspond respectively to the product and covariant derivative of gauge invariant operators. In fact, any gauge invariant tensor is built using the two operations just described.

In order to prove the lemma we first show that it holds for $n=0$. Then $\eta_{a b}$ is an antisymmetric tensor and $\lambda^{a} \star \lambda^{b} \eta_{a b}=\frac{1}{2}\left[\lambda^{a}, \lambda^{b}\right] \eta_{a b} \rightarrow \frac{1}{2}\left[\lambda^{a}, \lambda^{b}\right] \eta_{a b}+$ $\frac{i}{2} \Delta^{a b} \eta_{a b}$. Now suppose that we have proved the result for $\eta_{1 \cdots n}$ and $\nu_{1} \cdots m$. Then we must show that $\bar{\Delta}(\eta \nu)=(\bar{\Delta} \eta) \nu+\eta(\bar{\Delta} \nu)$. This is easily done, since

$$
\begin{aligned}
& \bar{\Delta}(\eta \nu)-(\bar{\Delta} \eta) \nu-\eta(\bar{\Delta} \nu) \propto \\
& \Delta^{a b}\left(\eta_{a, 1, \cdots, n-1}+\eta_{1, a, \cdots, n-1}+\cdots+\eta_{1, \cdots, n-1, a}\right) \cdot \\
& \cdot\left(\nu_{b, n \cdots, n+m-2}+\nu_{n, b, \cdots, n+m-2}+\cdots+\nu_{n, \cdots, n+m-2, b}\right)
\end{aligned}
$$

which vanishes since $\eta$ and $\nu$ are gauge invariant. Finally me must show that, given a generic $d_{a}$, one has $\bar{\Delta}(d \eta)=d(\bar{\Delta} \eta)$. Again this is easy to show using the gauge invariance of $\eta$, since

$$
\bar{\Delta}(d \eta)-d(\bar{\Delta} \eta) \propto \Delta^{a b} d_{a}\left(\eta_{b, 1, \cdots, n}+\eta_{1, b, \cdots, n}+\cdots+\eta_{1, \cdots, n, b}\right)=0 .
$$

This concludes the proof, since any gauge invariant operator is a product of covariant derivatives of the field strength. 
Lemma 7. (Section 3.7) Let $\eta_{1 \cdots n+2}$ be gauge invariant and let $\tau_{1 \cdots n+2}$ be the associated cyclic gauge invariant tensor. Let $\Delta^{a b}$ be antisymmetric, and define

$$
g_{1 \cdots n}=\frac{1}{n}\left(\bar{\Delta} \eta_{1 \cdots n}+\operatorname{cyc}_{1 \cdots n}\right)
$$

where $\bar{\Delta} \eta$ is given by expression (15). The tensor $g$ is then uniquely a function of $\tau$, and is explicitly given by the expression

$$
-\frac{i}{2}\left(\frac{n+2}{n^{2}}\right) \Delta^{a b}\left[n \tau_{1 \cdots n a b}+(n-1) \tau_{1 \cdots a n b}+\cdots+0 \cdot \tau_{a 1 \cdots n b}\right]+\operatorname{cyc}_{1 \cdots n}
$$

which will be denoted by $\bar{\Delta} \tau$.

PROOF. Define the following tensors

$$
\begin{aligned}
k_{1 \cdots n a b} & =\eta_{1 \cdots n a b}+\operatorname{cyc}_{1 \cdots n} \\
k_{1 \cdots a n b} & =\eta_{1 \cdots a n b}+\operatorname{cyc}_{1 \cdots n} \\
k_{1 \cdots n-1, b a, n} & =\eta_{1 \cdots n-1, b, a, n}+\operatorname{cyc}_{1 \cdots n}
\end{aligned}
$$

which have two selected indices $a, b$ and are cyclically symmetric in the other indices $1, \cdots, n$. It is clear that

$$
\begin{aligned}
\tau_{1 \cdots n a b}+\operatorname{cyc}_{1 \cdots n} & =\frac{1}{n+2}\left(k_{1 \cdots n a b}+\operatorname{cyc}_{1 \cdots n a b}\right) \\
\tau_{1 \cdots a n b}+\operatorname{cyc}_{1 \cdots n} & =\frac{1}{n+2}\left(k_{1 \cdots a n b}+\operatorname{cyc}_{1 \cdots a n b}\right)
\end{aligned}
$$

We can also express equation (64) for $g$ in terms of the tensors $k$ as

$$
\begin{aligned}
g_{1 \cdots n} & =-\frac{i}{2 n^{2}} \Delta^{a b} J_{1 \cdots n a b} \\
J_{1 \cdots n a b} & =n k_{1 \cdots n a b}+n k_{1 \cdots a n b}+\cdots,
\end{aligned}
$$

where, as in $\bar{\Delta} \eta$, the indices $a, b$ are in all possible positions with $a$ preceding $b$. We then want to prove that the above expression is equal to (65), which we can also write in terms of the tensors $k$ as follows

$$
-\frac{i}{2 n^{2}} \Delta^{a b} \widetilde{J}_{1 \cdots n a b}
$$


with

$$
\begin{aligned}
\widetilde{J}_{1 \cdots n a b}= & n\left(k_{1 \cdots n a b}+\operatorname{cyc}_{1 \cdots n a b}\right) \\
& +(n-1)\left(k_{1 \cdots a n b}+\operatorname{cyc}_{1 \cdots a n b}\right) \\
& +\cdots \\
& +0 \cdot\left(k_{a 1 \cdots n b}+\operatorname{cyc}_{a 1 \cdots n b}\right)
\end{aligned}
$$

We will actually prove that $J_{1 \cdots n a b}=\widetilde{J}_{1 \cdots n a b}$. In order to do this, we use the gauge invariance of the tensor $\eta$, which implies various linear relations among the tensors $k$

$$
\begin{aligned}
0= & k_{a b 1 \cdots n}+k_{b a 1 \cdots n}+k_{b 1 a \cdots n}+\cdots+k_{b 1 \cdots n a} \\
0= & k_{a 1 b \cdots n}+k_{1 a b \cdots n}+k_{1 b a \cdots n}+\cdots+k_{1 b 2 \cdots n a} \\
& \cdots
\end{aligned}
$$

We then need to prove that the difference $J-\widetilde{J}$ can be written as a linear combination of the above equations.

In order to write an efficient and clear proof, we will concentrate, from now on, on the case $n=4$. The proof in the general case is absolutely identical, but the added notation would obscure the result without adding new ideas to the ones already contained in the special case discussed below. We introduce the following compact notation

$$
\begin{aligned}
& k_{1234 a b} \rightarrow \cdot \cdot \cdot \cdot \cdot a b \\
& k_{123 a 4 b} \rightarrow \cdot \cdot \cdot a \cdot b
\end{aligned}
$$

where the dots . . . indicate the indices $1,2,3,4$. We then arrange all 
the possible tensors $k$ in the following tableau

\begin{tabular}{|cccccc|cccccc|}
\hline$\cdot$ & $\cdot$ & $\cdot$ & $\cdot$ & $a$ & $b$ & $\cdot$ & $\cdot$ & $\cdot$ & $\cdot$ & $b$ & $a$ \\
$\cdot$ & $\cdot$ & $\cdot$ & $a$ & $b$ & $\cdot$ & $\cdot$ & $\cdot$ & $\cdot$ & $b$ & $a$ & $\cdot$ \\
$\cdot$ & $\cdot$ & $a$ & $b$ & $\cdot$ & $\cdot$ & $\cdot$ & $\cdot$ & $b$ & $a$ & $\cdot$ & $\cdot$ \\
$\cdot$ & $a$ & $b$ & $\cdot$ & $\cdot$ & $\cdot$ & $\cdot$ & $b$ & $a$ & $\cdot$ & $\cdot$ & $\cdot$ \\
$a$ & $b$ & $\cdot$ & $\cdot$ & $\cdot$ & $\cdot$ & $b$ & $a$ & $\cdot$ & $\cdot$ & $\cdot$ & $\cdot$ \\
\hline$\cdot$ & $\cdot$ & $\cdot$ & $a$ & $\cdot$ & $b$ & $\cdot$ & $\cdot$ & $\cdot$ & $b$ & $\cdot$ & $a$ \\
$\cdot$ & $\cdot$ & $a$ & $\cdot$ & $b$ & $\cdot$ & $\cdot$ & $\cdot$ & $b$ & $\cdot$ & $a$ & $\cdot$ \\
$\cdot$ & $a$ & $\cdot$ & $b$ & $\cdot$ & $\cdot$ & $\cdot$ & $b$ & $\cdot$ & $a$ & $\cdot$ & $\cdot$ \\
$a$ & $\cdot$ & $b$ & $\cdot$ & $\cdot$ & $\cdot$ & $b$ & $\cdot$ & $a$ & $\cdot$ & $\cdot$ & $\cdot$ \\
\hline$\cdot$ & $\cdot$ & $a$ & $\cdot$ & $\cdot$ & $b$ & $\cdot$ & $\cdot$ & $b$ & $\cdot$ & $\cdot$ & $a$ \\
$\cdot$ & $a$ & $\cdot$ & $\cdot$ & $b$ & $\cdot$ & $\cdot$ & $b$ & $\cdot$ & $\cdot$ & $a$ & $\cdot$ \\
$a$ & $\cdot$ & $\cdot$ & $b$ & $\cdot$ & $\cdot$ & $b$ & $\cdot$ & $\cdot$ & $a$ & $\cdot$ & $\cdot$ \\
\hline$\cdot$ & $a$ & $\cdot$ & $\cdot$ & $\cdot$ & $b$ & $\cdot$ & $b$ & $\cdot$ & $\cdot$ & $\cdot$ & $a$ \\
$a$ & $\cdot$ & $\cdot$ & $\cdot$ & $b$ & $\cdot$ & $b$ & $\cdot$ & $\cdot$ & $\cdot$ & $a$ & $\cdot$ \\
\hline$a$ & $\cdot$ & $\cdot$ & $\cdot$ & $\cdot$ & $b$ & $b$ & $\cdot$ & $\cdot$ & $\cdot$ & $\cdot$ & $a$ \\
\hline
\end{tabular}

which has on the left all terms with $a$ preceding $b$, and on the right all terms with $b$ before $a$. From top to bottom, the terms are, on the other hand, arranged in groups with a fixed number of indices between $a$ and $b$. We then denote any linear combination of the tensors $k$ with a horizontal box of coefficients

\begin{tabular}{|c|c|c|c|c|}
\hline$a_{1} a_{2} a_{3} a_{4} a_{5}$ & $b_{1} b_{2} b_{3} b_{4}$ & $c_{1} c_{2} c_{3}$ & $d_{1} d_{2}$ & $e_{1}$ \\
\hline$f_{1} f_{2} f_{3} f_{4} f_{5}$ & $g_{1} g_{2} g_{3} g_{4}$ & $h_{1} h_{2} h_{3}$ & $i_{1} i_{2}$ & $j_{1}$ \\
\hline
\end{tabular}

where the top line corresponds to the coefficients of the left column in the tableau, and the bottom line to the right column (the above box is then a compact notation for the sum $\left.a_{1} k_{1234 a b}+a_{2} k_{123 a 4 b}+\cdots+f_{1} k_{1234 b a}+\cdots\right)$. The main statement which we want to prove can now be compactly written as

\begin{tabular}{|l|l|l|l|l|}
\hline 44444 & 4444 & 444 & 44 & 4 \\
\hline 00000 & 0000 & 000 & 00 & 0 \\
\hline
\end{tabular}$=$\begin{tabular}{|l|l|l|l|l|}
\hline 44444 & 3333 & 222 & 11 & 0 \\
\hline 00000 & 1111 & 222 & 33 & 4 \\
\hline
\end{tabular}

In fact the LHS above is nothing but $J$. To show that the RHS is $\widetilde{J}$ we just note that

$$
\begin{aligned}
& k_{1 \cdots n a b}+\mathrm{cyc}_{1 \cdots n a b}=\begin{array}{|l|l|l|l|l|}
\hline 11111 & 0000 & 000 & 00 & 0 \\
\hline 00000 & 0000 & 000 & 00 & 1 \\
\hline
\end{array} \\
& k_{1 \cdots a n b}+\mathrm{cyc}_{1 \cdots a n b}=\begin{array}{|l|l|l|l|l|}
\hline 00000 & 1111 & 000 & 00 & 0 \\
\hline 00000 & 0000 & 000 & 11 & 0 \\
\hline
\end{array}
\end{aligned}
$$


To prove the equality (67) we use the linear relations (66), which can also be compactly rewritten as

$$
a_{i}=b_{j}=0
$$

where

$$
\begin{aligned}
& a_{1}=\begin{array}{|l|l|l|l|l|}
\hline 10000 & 1000 & 100 & 10 & 1 \\
\hline 10000 & 0000 & 000 & 00 & 0 \\
\hline
\end{array} \\
& b_{1}=\begin{array}{|l|l|l|l|l|}
\hline 00001 & 0001 & 001 & 01 & 1 \\
\hline 00001 & 0000 & 000 & 00 & 0 \\
\hline
\end{array} \\
& a_{2}=\begin{array}{|l|l|l|l|l|}
\hline 01000 & 0100 & 010 & 01 & 0 \\
\hline 01000 & 1000 & 000 & 00 & 0 \\
\hline
\end{array} \quad b_{2}=\begin{array}{|l|l|l|l|l|l|l|}
\hline 00010 & 0010 & 010 & 10 & 0 \\
\hline 00010 & 0001 & 000 & 00 & 0 \\
\hline
\end{array} \\
& a_{3}=\begin{array}{|l|l|l|l|l|}
\hline 00100 & 0010 & 001 & 00 & 0 \\
\hline 00100 & 0100 & 100 & 00 & 0 \\
\hline
\end{array} \quad b_{3}=\begin{array}{|l|l|l|l|l|l|}
\hline 00100 & 0100 & 100 & 00 & 0 \\
\hline 00100 & 0010 & 001 & 00 & 0 \\
\hline
\end{array} \\
& a_{4}=\begin{array}{|l|l|l|l|l|}
\hline 00010 & 0001 & 000 & 00 & 0 \\
\hline 00010 & 0010 & 010 & 10 & 0 \\
\hline
\end{array} \quad b_{4}=\begin{array}{|l|l|l|l|l|l|l|}
\hline 01000 & 1000 & 000 & 00 & 0 \\
\hline 01000 & 0100 & 010 & 01 & 0 \\
\hline
\end{array} \\
& a_{5}=\begin{array}{|l|l|l|l|l|}
\hline 00001 & 0000 & 000 & 00 & 0 \\
\hline 00001 & 0001 & 001 & 01 & 1 \\
\hline
\end{array} \quad b_{5}=\begin{array}{|l|l|l|l|l|l|}
\hline 10000 & 0000 & 000 & 00 & 0 \\
\hline 10000 & 1000 & 100 & 10 & 1 \\
\hline
\end{array}
\end{aligned}
$$

Summing either all the $a$ 's or all the $b$ 's we first of all obtain the following interesting identity

\begin{tabular}{|l|l|l|l|l|}
\hline 11111 & 1111 & 111 & 11 & 1 \\
\hline 11111 & 1111 & 111 & 11 & 1 \\
\hline
\end{tabular}

Combining the above equation with (67) we can then reduce the statement of the lemma to the following equality

\begin{tabular}{|l|l|l|l|l|}
\hline 44444 & 5555 & 666 & 77 & 8 \\
\hline 44444 & 3333 & 222 & 11 & 0 \\
\hline
\end{tabular}

The LHS above is nothing but

$$
4\left(a_{1}+b_{1}\right)+3\left(a_{2}+b_{2}\right)+2\left(a_{3}+b_{3}\right)+1\left(a_{4}+b_{4}\right)+0\left(a_{5}+b_{5}\right),
$$

and therefore the proof is complete.

Remark 3. Using the box (68) we can show that the expression

$$
\left[n \tau_{1 \cdots n a b}+(n-1) \tau_{1 \cdots a n b}+\cdots+0 \cdot \tau_{a 1 \cdots n b}\right]+\operatorname{cyc}_{1 \cdots n}
$$

in (65) is antisymmetric in $a$ and $b$.

Lemma 8. (Section 5.1) Let $\tau_{1 \cdots n}$ be a cyclic gauge invariant tensor, and let $\Delta_{a b}$ be an arbitrary invertible antisymmetric matrix with inverse $\Delta^{a b}$. Then

$$
[\bar{\Delta}, \Delta] \tau=\left(n-\frac{1}{2} D\right) \tau
$$


PROOF. First we recall the expression for $\Delta \tau$, which is given by

$$
(\Delta \tau)_{1 \cdots n+2}=\frac{i}{2}\left(\frac{n}{n+2}\right)\left(\Delta_{12} \tau_{345 \cdots n+2}-\Delta_{13} \tau_{245 \cdots n+2}\right)+\operatorname{cyc}_{1 \cdots n+2} .
$$

We then concentrate on the expression $\bar{\Delta} \Delta \tau$ by recalling, first of all, that the operation $\bar{\Delta}$ on $\Delta \tau$ consists of a contraction of two indices of the tensor $\Delta \tau$ with the antisymmetric tensor $\Delta^{a b}$. It is then clear, since $\Delta \tau$ itself is built from the tensors $\tau_{1 \cdots n}$ and $\Delta_{a b}$, that

$$
\bar{\Delta} \Delta \tau=A+B+C,
$$

where $A$ contains the terms where $\Delta^{a b}$ is contracted uniquely with $\Delta_{a b}$ and $B$ contains terms in which the two indices in $\Delta^{a b}$ are contracted one with $\Delta_{a b}$ and one with $\tau_{1 \cdots n}$. Finally $C$ consists of the remaining terms, with contractions of $\Delta^{a b}$ only with $\tau_{1 \cdots n}$. We will prove in the sequel that

$$
\begin{aligned}
& A=-\frac{1}{2} D \tau \\
& B=n \tau \\
& C=\Delta \bar{\Delta} \tau,
\end{aligned}
$$

thus proving the lemma.

Let me start by concentrating on the terms in $A$. First recall the expression for $\bar{\Delta} \Delta \tau$

$$
\bar{\Delta} \Delta \tau=-\frac{i}{2}\left(\frac{n+2}{n^{2}}\right) \Delta^{a b}\left[n \cdot(\Delta \tau)_{1 \cdots n a b}+\cdots+1 \cdot(\Delta \tau)_{1 a 2 \cdots n b}\right]+\operatorname{cyc}_{1 \cdots n}
$$

The only terms in the above equation which contribute to $A$ are the first, second and last within the square bracket. In particular the first term reads

$$
\begin{aligned}
& -\frac{i}{2}\left(\frac{n+2}{n}\right) \Delta^{a b}(\Delta \tau)_{1 \cdots n a b}+\operatorname{cyc}_{1 \cdots n} \\
= & \frac{1}{4} \Delta^{a b}\left[\left(\Delta_{12} \tau_{3 \cdots n a b}-\Delta_{13} \tau_{24 \cdots n a b}\right)+\operatorname{cyc}_{1 \cdots n a b}\right]+\operatorname{cyc}_{1 \cdots n} \\
= & \frac{1}{4} \Delta^{a b} \Delta_{a b} \tau_{1 \cdots n}+\operatorname{cyc}_{1 \cdots n}+\text { terms not in } A \\
= & -n \frac{D}{4} \tau_{1 \cdots n}+\text { terms not in } A,
\end{aligned}
$$

where we have used that

$$
\Delta_{a b} \Delta^{a b}=-D
$$


and that $\tau$ is cyclic. Similarly, the second term is given by

$$
\begin{aligned}
& -\frac{i}{2}\left(\frac{n+2}{n^{2}}\right)(n-1) \Delta^{a b}(\Delta \tau)_{1 \cdots a n b}+\operatorname{cyc}_{1 \cdots n} \\
= & \left(\frac{n-1}{4 n}\right) \Delta^{a b}\left[\left(\Delta_{12} \tau_{3 \cdots a n b}-\Delta_{13} \tau_{24 \cdots a n b}\right)+\operatorname{cyc}_{1 \cdots a n b}\right]+\operatorname{cyc}_{1 \cdots n} \\
= & (n-1) \frac{D}{4} \tau_{1 \cdots n}+\text { terms not in } A .
\end{aligned}
$$

Finally the last term

$$
\begin{aligned}
& -\frac{i}{2}\left(\frac{n+2}{n^{2}}\right) \Delta^{a b}(\Delta \tau)_{1 a 2 \cdots n b}+\operatorname{cyc}_{1 \cdots n} \\
= & \frac{1}{4 n} \Delta^{a b}\left[\left(\Delta_{1 a} \tau_{2 \cdots n b}-\Delta_{12} \tau_{a 3 \cdots n b}\right)+\operatorname{cyc}_{1 a 2 \cdots n b}\right]+\operatorname{cyc}_{1 \cdots n} \\
= & -\frac{D}{4} \tau_{1 \cdots n}+\text { terms not in } A
\end{aligned}
$$

Summing the three contributions we obtain

$$
A=-\frac{D}{2} \tau
$$

as was to be shown.

We now move to the analysis of the terms in $B$. Again we consider equation (69), and we focus once again on the first term in the square brackets

$$
\frac{1}{4} \Delta^{a b}\left[\left(\Delta_{12} \tau_{3 \cdots n a b}-\Delta_{13} \tau_{24 \cdots n a b}\right)+\operatorname{cyc}_{1 \cdots n a b}\right]+\operatorname{cyc}_{1 \cdots n}
$$

We concentrate on the terms in $B$, with $\Delta^{a b}$ contracted both with $\Delta_{a b}$ and with $\tau_{1 \cdots n}$

$$
\begin{aligned}
& \frac{1}{4} \Delta^{a b}\left[\Delta_{n a} \tau_{b 1 \cdots n-1}+\Delta_{b 1} \tau_{2 \cdots n a}\right]+\operatorname{cyc}_{1 \cdots n} \\
& -\frac{1}{4} \Delta^{a b}\left[\Delta_{a 1} \tau_{b 2 \cdots n}+\Delta_{n b} \tau_{a 1 \cdots n-1}+\Delta_{n-1, a} \tau_{n b 1 \cdots n-2}+\Delta_{b 2} \tau_{13 \cdots n a}\right] \\
& +\operatorname{cyc}_{1 \cdots n} \\
= & \frac{1}{4 n}\left(4 n \tau_{1 \cdots n}-n \tau_{213 \cdots n}-n \tau_{23 \cdots 1 n}\right)+\operatorname{cyc}_{1 \cdots n}
\end{aligned}
$$

The second term in (69)

$$
\left(\frac{n-1}{4 n}\right) \Delta^{a b}\left[\left(\Delta_{12} \tau_{3 \cdots a n b}-\Delta_{13} \tau_{24 \cdots a n b}\right)+\operatorname{cyc}_{1 \cdots a n b}\right]+\operatorname{cyc}_{1 \cdots n}
$$


gives as contribution to $B$

$$
\begin{aligned}
& \left(\frac{n-1}{4 n}\right) \Delta^{a b}\left[\Delta_{b 1} \tau_{23 \cdots a n}+\Delta_{n b} \tau_{1 \cdots n-1 a}+\Delta_{n-1, a} \tau_{n b 1 \cdots n-2}+\Delta_{a n} \tau_{b 1 \cdots n-1}\right] \\
& -\left(\frac{n-1}{4 n}\right) \Delta^{a b}\left[\Delta_{b 2} \tau_{13 \cdots a n}+\Delta_{n-2, a} \tau_{n-1, n, b, 1 \cdots, n-3}\right]+\operatorname{cyc}_{1 \cdots n} \\
& =\left(\frac{n-1}{4 n}\right)\left[\tau_{213 \cdots n}+\tau_{23 \cdots 1 n}-\tau_{231 \cdots n}-\tau_{23 \cdots, 1, n-1, n}-2 \tau_{1 \cdots n}\right]+ \\
& +\operatorname{cyc}_{1 \cdots n}
\end{aligned}
$$

In order to write equations in a compact form let us introduce some notation. We explain the notation in the case $n=4$, but then we continue the proof in a general setting. We wish to consider tensors $\tau$... with the indices given by $2,3,4$ in increasing order, and with the index 1 in a given position. For example, if the index 1 is in the 3rd position we are considering the tensor $\tau_{2314}$, which we will denote with the following box

$$
\tau_{2314} \rightarrow \begin{array}{l|l|l|l|}
\hline 0 & 0 & 1 & 0 \\
\hline
\end{array}
$$

Moreover, linear combinations of the various tensors will also be denoted by a single box in the following obvious way

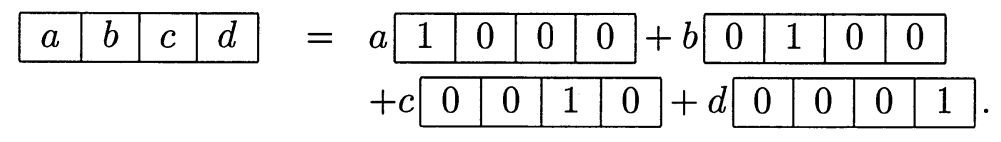

Let us now return to the general proof, by first showing some simple properties of the box just introduced. Cyclicity of the tensor $\tau$ implies that

$$
\begin{array}{|l|l|l|l|l|}
\hline 1 & 0 & \cdots & 0 & 0 \\
\hline
\end{array}=\begin{array}{l|l|l|l|l|}
\hline 0 & 0 & \cdots & 0 & 1 \\
\hline
\end{array}
$$

Moreover, cyclic gauge invariance of $\tau$ implies the two equivalent identities

$$
\begin{array}{|l|l|l|l|l|}
\hline 1 & 1 & \cdots & 1 & 0 \\
\hline
\end{array}
$$

which can be summed to obtain

$$
\begin{array}{|l|l|l|l|l|}
\hline 1 & 2 & \cdots & 2 & 1 \\
\hline
\end{array}
$$

We can use this notation to compactly rewrite the first two terms in $\bar{\Delta} \Delta \tau$ given by equations (70) and (71). They now read

$$
\begin{array}{|l|l|l|l|l|l|l|}
\hline & 1 \\
\hline 4 n & -n & 0 & \cdots & 0 & -n & 2 n \\
\hline
\end{array}
$$


and

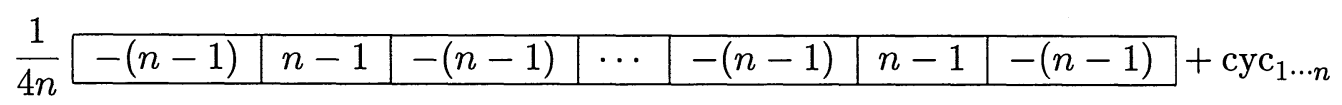

We notice that both terms are represented by boxes which are symmetric about a vertical axis of symmetry. Moreover we can do computations similar to the ones above to convince ourselves that the $(k+1)$-th term in $\bar{\Delta} \Delta \tau$ is given by the following box (we just show the left side of the box, since the right side is just its mirror copy)

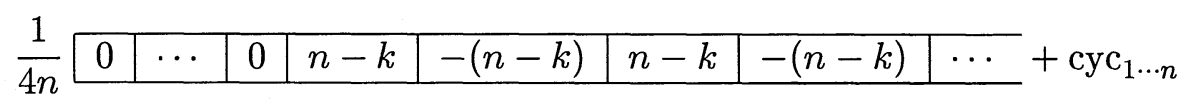

with $k-2$ zeros on the left before the term $n-k$. Writing at once all the contributions to $B$ we get the following tableau

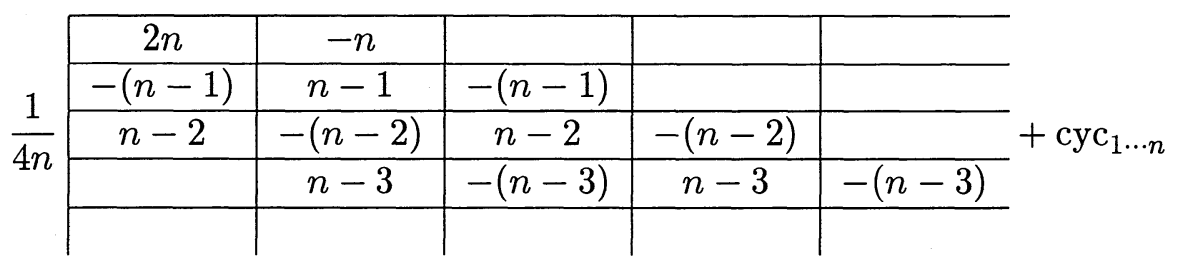

where we must sum the coefficients in each column. The result is then

$$
\begin{aligned}
& \begin{array}{ll|l|l|l|l|l|l|}
\frac{1}{4 n} & 2 n-1 & -2 & -2 & \cdots & -2 & -2 & 2 n-1 \\
\hline
\end{array} \\
& =\frac{1}{2} \begin{array}{|l|l|l|l|l|}
\hline 1 & 0 & \cdots & 0 & 1 \\
\hline
\end{array}
\end{aligned}
$$

where we have used equations (73) and (72). Going back to the usual tensor notation we have then obtained

$$
B=\tau_{1 \cdots n}+\operatorname{cyc}_{1 \cdots n}=n \tau .
$$

We conclude the proof by showing that $C=\Delta \bar{\Delta} \tau$. Consider again the first term in $\bar{\Delta} \Delta \tau$

$$
\frac{1}{4} \Delta^{a b}\left[\left(\Delta_{12} \tau_{3 \cdots n a b}-\Delta_{13} \tau_{24 \cdots n a b}\right)+\operatorname{cyc}_{1 \cdots n a b}\right]+\operatorname{cyc}_{1 \cdots n}
$$

and concentrate on the terms which contain $\tau_{\ldots a b}-i . e$. terms for which the indices in $\tau$ contain $a$ just before $b$ -

$$
\begin{aligned}
& \frac{1}{4}\left(\Delta_{12} \Delta^{a b}\left(\tau_{3 \cdots n a b}+\operatorname{cyc}_{3 \cdots n}\right)+\operatorname{cyc}_{1 \cdots n}\right) \\
& -\frac{1}{4}\left(\Delta_{13} \Delta^{a b}\left(\tau_{24 \cdots n a b}+\operatorname{cyc}_{24 \cdots n}\right)+\operatorname{cyc}_{1 \cdots n}\right) \\
& +\frac{1}{4}\left(\Delta_{12} \Delta^{a b} \tau_{3 \cdots n a b}+\operatorname{cyc}_{1 \cdots n}\right)
\end{aligned}
$$


Terms with $\tau_{\ldots a b}$ are also contained in the second and third contribution to $\Delta \bar{\Delta} \tau$ in $(69)$

$$
\begin{aligned}
& \left(\frac{n-1}{4 n}\right) \Delta^{a b}\left[\left(\Delta_{12} \tau_{3 \cdots a n b}-\Delta_{13} \tau_{24 \cdots a n b}\right)+\operatorname{cyc}_{1 \cdots a n b}\right]+\operatorname{cyc}_{1 \cdots n} \\
& \left(\frac{n-2}{4 n}\right) \Delta^{a b}\left[\left(\Delta_{12} \tau_{3 \cdots a, n-1, n b}-\Delta_{13} \tau_{24 \cdots a, n-1, n b}\right)+\operatorname{cyc}_{1 \cdots a, n-1, n, b}\right]+\operatorname{cyc}_{1 \cdots n}
\end{aligned}
$$

They are respectively

$$
-2\left(\frac{n-1}{4 n}\right)\left(\Delta_{12} \Delta^{a b} \tau_{3 \cdots n a b}+\operatorname{cyc}_{1 \cdots n}\right)
$$

and

$$
\left(\frac{n-2}{4 n}\right)\left(\Delta_{12} \Delta^{a b} \tau_{3 \cdots n a b}+\operatorname{cyc}_{1 \cdots n}\right) .
$$

Summing equations (74),(75) and (76) we then obtain (the last line in (74) is canceled by (75) and (76))

$$
\begin{aligned}
& \frac{1}{4}\left(\Delta_{12} \Delta^{a b}\left(\tau_{3 \cdots n a b}+\operatorname{cyc}_{3 \cdots n}\right)+\operatorname{cyc}_{1 \cdots n}\right) \\
& -\frac{1}{4}\left(\Delta_{13} \Delta^{a b}\left(\tau_{24 \cdots n a b}+\operatorname{cyc}_{24 \cdots n}\right)+\operatorname{cyc}_{1 \cdots n}\right) .
\end{aligned}
$$

But this is exactly the result which is obtained by computing terms proportional to $\tau \ldots a b$ in $\Delta \bar{\Delta} \tau$, since

$$
\Delta \bar{\Delta} \tau=\frac{i}{2}\left(\frac{n-2}{n}\right)\left[\Delta_{12}(\bar{\Delta} \tau)_{34 \cdots n}-\Delta_{13}(\bar{\Delta} \tau)_{24 \cdots n}+\operatorname{cyc}_{1 \cdots n}\right]
$$

and, if we concentrate on terms of the form $\tau_{\ldots a b}$,

$$
(\bar{\Delta} \tau)_{34 \cdots n}=-\frac{i}{2}\left(\frac{n}{n-2}\right) \Delta^{a b}\left(\tau_{3 \cdots n a b}+\operatorname{cyc}_{3 \cdots n}\right) .
$$

Similar arguments can be used for terms proportional to $\tau_{\ldots a \cdots b}$, with different number of indices between $a$ and $b$. We have then shown that $C=\Delta \bar{\Delta} \tau$, thus completing the proof.

Lemma 9. (Section 6.2) If $F=[Z, \bar{Z}]$, then

$$
\Delta F^{3}=2 F^{4}+\frac{1}{2} F[D F, \bar{D} F]
$$


PROOF. We compute the word $w \in W_{4}$

$$
w=\Delta F^{3} .
$$

First we have that

$$
w=3 F^{2}(\Delta F)=\frac{3}{2}\left(F^{4}+F[F Z, F \bar{Z}]\right) .
$$

We then use cyclicity to show that

$$
\begin{aligned}
F[F Z, F \bar{Z}] & =F^{4}+F^{2}[F, \bar{Z}] Z+F^{2}[Z, F] \bar{Z} \\
& =F^{4}+Z F^{2}[F, \bar{Z}]+\bar{Z} F^{2}[Z, F] \\
& =F^{4}+F^{2} \bar{Z}[Z, F]+\bar{Z} F^{2}[Z, F]
\end{aligned}
$$

and therefore that

$$
w=3 F^{4}+\frac{3}{2}\left(F^{2} \bar{Z}[Z, F]+\bar{Z} F^{2}[Z, F]\right) .
$$

Now, since

$$
F^{2} \bar{Z}[Z, F]=\left[F^{2} \bar{Z}, Z\right] F=-F^{4}-\bar{Z} F^{2}[Z, F]-F \bar{Z} F[Z, F]
$$

and since

$$
\begin{aligned}
-F \bar{Z} F[Z, F] & =F D F \bar{D} F-\bar{Z} F^{2}[Z, F] \\
& =-F \bar{D} F D F-F^{2} \bar{Z}[Z, F] \\
& =\frac{1}{2}\left(F[D F, \bar{D} F]-\bar{Z} F^{2}[Z, F]-F^{2} \bar{Z}[Z, F]\right),
\end{aligned}
$$

we obtain that

$$
\frac{3}{2}\left(F^{2} \bar{Z}[Z, F]+\bar{Z} F^{2}[Z, F]\right)=-F^{4}+\frac{1}{2} F[D F, \bar{D} F] .
$$

The above fact, together with (77), concludes the proof of the lemma. 


\section{References}

[1] N. Seiberg and E. Witten, "String theory and noncommutative geometry", JHEP 9909, 032 (1999), hep-th/9908142.

[2] V. Schomerus, "D-branes and deformation quantization", JHEP 9906, 030 (1999), hep-th/9903205.

[3] A. A. Tseytlin, "Born-Infeld action, supersymmetry and string theory", hep-th/9908105.

[4] O. D. Andreev and A. A. Tseytlin, "Partition Function Representation For The Open Superstring Effective Action: Cancellation Of Mobius Infinities And Derivative Corrections To Born-Infeld Lagrangian", Nucl. Phys. B 311, 205 (1988).

[5] A. A. Tseytlin, "On non-abelian generalisation of the Born-Infeld action in string theory", Nucl. Phys. B501, 41 (1997), hep-th/9701125.

[6] A. A. Tseytlin, "Vector Field Effective Action In The Open Superstring Theory", Nucl. Phys. B276, 391 (1986).

[7] A. Cattaneo and G. Felder, "A Path Integral Approach to the Kontsevich Quantization Formula", math.QA/9902090.

[8] B. Jurco and P. Schupp, "Noncommutative Yang-Mills from equivalence of star products", hep-th/0001032.

[9] M. Kontsevich, "Deformation quantization of Poisson manifolds", q-alg/99709040.

[10] Y. Okawa, "Derivative corrections to Dirac-Born-Infeld Lagrangian and non-commutative gauge theory", hep-th/9909132.

S. Terashima, "The non-Abelian Born-Infeld action and noncommutative gauge theory", hep-th/0006058.

Y. Okawa and S. Terashima, "Constraints on effective Lagrangian of D-branes from non-commutative gauge theory", hep-th/0002194.

S. Terashima, "On the equivalence between noncommutative and ordinary gauge theories," hep-th/0001111.

[11] L. Cornalba, "Corrections to the Abelian Born-Infeld Action Arising from Noncommutative Geometry", hep-th/9912293.

L. Cornalba, "D-brane physics and noncommutative Yang-Mills theory", hep-th/9909081. 
L. Cornalba and R. Schiappa, "Matrix theory star products from the Born-Infeld action", hep-th/9907211.

[12] C. G. Callan, C. Lovelace, C. R. Nappi and S. A. Yost, "Loop Corrections To Conformal Invariance For Type 1 Superstrings", Phys. Lett. B206, 41 (1988).

C. G. Callan, C. Lovelace, C. R. Nappi and S. A. Yost, "String Loop Corrections To Beta Functions", Nucl. Phys. B288, 525 (1987).

[13] L. Cornalba and W. I. Taylor, "Holomorphic curves from matrices", Nucl. Phys. B536, 513 (1998), hep-th/9807060.

L. Cornalba, "Matrix representations of holomorphic curves on $\mathrm{T}(4)$ ", hep-th/9812184.

[14] R. G. Leigh, "Dirac-Born-Infeld Action From Dirichlet Sigma Model", Mod. Phys. Lett. A4, 2767 (1989).

[15] O. Andreev and H. Dorn, "On open string sigma-model and noncommutative gauge fields", Phys. Lett. B476, 402 (2000)

[16] L. Cornalba, "T-duality without T-duality", in preparation.

[17] W. I. Taylor and M. Van Raamsdonk, "Multiple D0-branes in weakly curved backgrounds", Nucl. Phys. B558, 63 (1999), hep--th/9904095.

W. I. Taylor and M. Van Raamsdonk, "Multiple Dp-branes in weak background fields", Nucl. Phys. B573, 703 (2000), hep--th/9910052.

[18] R. C. Myers, "Dielectric-branes", JHEP 9912, 022 (1999), hep-th/9910053.

[19] A. Hashimoto and W. I. Taylor, "Fluctuation spectra of tilted and intersecting D-branes from the Born-Infeld action," Nucl. Phys. B503, 193 (1997), hep--th/9703217.

[20] C. P. Bachas, P. Bain and M. B. Green, "Curvature terms in Dbrane actions and their M-theory origin", JHEP 9905, 011 (1999), hep--th/9903210.

[21] Y. Kitazawa, "Effective Lagrangian For Open Superstring From Five Point Function", Nucl. Phys. B289, 599 (1987). 